ARTICLE

\title{
Single-cell profiling of human subventricular zone progenitors identifies SFRP1 as a target to re-activate progenitors
}

Vanessa Donega (1) ${ }^{1 凶}$, Astrid T. van der Geest (1D ${ }^{1}$, Jacqueline A. Sluijs ${ }^{1}$, Roland E. van Dijk ${ }^{1}$, Chi Chiu Wang (D) ${ }^{2,3}$, Onur Basak (i) ${ }^{1}$, R. Jeroen Pasterkamp $\mathbb{1}^{1} \&$ Elly M. Hol (i) ${ }^{1 凶}$

Following the decline of neurogenesis at birth, progenitors of the subventricular zone (SVZ) remain mostly in a quiescent state in the adult human brain. The mechanisms that regulate this quiescent state are still unclear. Here, we isolate $\mathrm{CD} 271^{+}$progenitors from the aged human SVZ for single-cell RNA sequencing analysis. Our transcriptome data reveal the identity of progenitors of the aged human SVZ as late oligodendrocyte progenitor cells. We identify the Wnt pathway antagonist SFRP1 as a possible signal that promotes quiescence of progenitors from the aged human SVZ. Administration of WAY-316606, a small molecule that inhibits SFRP1 function, stimulates activation of neural stem cells both in vitro and in vivo under homeostatic conditions. Our data unravel a possible mechanism through which progenitors of the adult human SVZ are maintained in a quiescent state and a potential target for stimulating progenitors to re-activate.

\footnotetext{
${ }^{1}$ Department of Translational Neuroscience, UMC Utrecht Brain Center, University Medical Center Utrecht, Utrecht University, 3584 CG Utrecht, The Netherlands. ${ }^{2}$ Department of Obstetrics and Gynecology, Li Ka Shing Institute of Health Sciences, School of Biomedical Sciences, and Chinese University of Hong Kong -Sichuan University Joint Laboratory in Reproductive Medicine, The Chinese University of Hong Kong, Shatin, NT, Hong Kong. ${ }^{3}$ Institute of Biochemistry, Charite-University Medicine Berlin, Charitéplatz 1, Berlin, Germany. ${ }^{\circledR}$ email: V.Donega@umcutrecht.nl; E.M.Hol-2@umcutrecht.nl
} 
n most mammals, neurogenesis in the dentate gyrus (DG) and subventricular zone (SVZ) continues during adulthood ${ }^{1}$. In rodents and non-human primates, new neurons generated in the SVZ migrate to the olfactory bulb. In humans, on the other hand, the addition of new neurons to the olfactory bulb is likely negligible ${ }^{1-5}$ and new neurons produced in the SVZ migrate to the neighboring striatum ${ }^{2}$. Growing evidence suggests that the decline in neurogenesis observed during aging in mammals is due to increased quiescence of neural stem cells (NSCs) and progenitors $^{6-8}$ (hereafter progenitors refers to both NSCs and progenitors).

Studies in rodents have shown that adult NSCs arise from a population of quiescent radial glial cells that accumulate embryonically 9,10 . Rather than being a static non-proliferating pool of cells, studies in rodents have demonstrated that they are a very dynamic population of cells that transit between proliferative and quiescent states ${ }^{11-13}$. With aging progenitors become less plastic and remain mainly quiescent, which prevents depletion of the progenitor pool ${ }^{7}$. The mechanisms that regulate quiescence of progenitors are just beginning to be unraveled ${ }^{6-8,14-23}$. With age, the germinal niches become less neurogenic due to increased inflammatory signals and Wnt pathway antagonists, and decreased activity of the Wnt pathway6,7,12,24-26. Despite the decrease in neurogenic function of the aged SVZ, adult progenitors are permissive to pharmacological or genetic approaches that stimulate their neurogenic potential7,27,28. Furthermore, progenitors were shown to exit quiescence and re-enter the cellcycle following ischemic injury in adult rodents ${ }^{12}$. Quiescent NSCs of the SVZ could be a potential source of stem cells for repair. However, the transcriptional programs that regulate quiescence of progenitors of the human brain are still unclear.

We have previously identified NGFR (i.e. CD271) as a marker expressed by progenitors of the aged human $\mathrm{SVZ}^{29-31}$. We showed that these cells form neurospheres and can differentiate into immature neurons and glia cells in vitro ${ }^{30}$. The present study assesses the molecular identity of NGFR-positive progenitors from the SVZ of the aged human brain at single-cell level and investigates a mechanism through which human progenitors could be maintained in a quiescent state. We identify the secreted frizzled-related protein-1 (SFRP1), an inhibitor of the Wnt signaling pathway, to be among genes whose expression changes over time. We demonstrate that inhibition of SFRP1 with a small molecule stimulates proliferation in vitro, in human iPSC-derived NSCs, and in vivo in early postnatal mice. Altogether, our work proposes a mechanism that maintains quiescence of progenitors of the human SVZ, which opens up future possibilities to stimulate NSCs of the human brain to promote repair.

\section{Results}

Characterization of the human SVZ at single-cell level. We have recently confirmed the progenitor identity of $\mathrm{NGFR}^{+}$(i.e. CD271) cells $^{31}$ from the human SVZ by assessing their transcriptome and proteome signature. To further characterize the dorsal SVZ of the aged human brain we performed single-cell RNA sequencing, on the freshly isolated dorsal SVZ from donors without known neurological or psychiatric disease $(n=3$, mean age $=88$; 2 females, aged 95 (504 cells, mean UMI per cell 3935, mean gene number per cell 1352) and 96 years (118 cells, mean UMI per cell 4181, mean gene number per cell 873); 1 male, aged 72 years (116 cells, mean UMI per cell 3991, mean gene number per cell 779)) (Supplementary Data 1). Progenitors, astrocytes, and microglia were isolated by fluorescently labeling the different populations for CD271 (progenitors) ${ }^{31}$, GLT-1 (astrocytes), and CD11b (microglia), followed by FACS (Supplementary Fig. 1a, b). We also sorted the negative fraction. We obtained the profile of
1074 cells from the SVZ of the aged human brain. After QCanalysis, 738 cells remained for further analysis (Supplementary Fig. 1c-h). We performed unbiased cluster analysis using the Louvain algorithm and the Uniform Manifold Approximation and Projection (UMAP) ${ }^{32,33}$ identifying seven clusters (Fig. 1a and Supplementary Fig. 2a). Cell types clustered based on biological cell type, rather than donor or technical artefacts (Supplementary Fig. 1d, e). We identified three microglia clusters, viz. Microglia 1, Microglia 2, and Microglia 3 as they expressed canonical microglia markers (e.g. CX3CR1 and AIF1) (Fig. 1a, b). These three clusters contained cells that were $\mathrm{CD}_{11} \mathrm{~b}^{+}$. We identified two clusters as progenitor clusters (i.e. Progenitors 1 and Progenitors 2), which expressed markers for progenitors (e.g. SOX2 and SOX10), and lacked expression of markers for ependymal cells (FOXJ1 and AQP4), radial glial cells (HOPX), or astrocytes (VIM, GFAP, and ALDH1A1) (Fig. 1c). These two clusters contained the cells that were sorted based on CD271 expression and some cells from the negative fraction (Supplementary Fig. 1e-h). They expressed the marker for early progenitor/astrocyte $C D 9^{12}$, but did not express markers for activated progenitors NES and $E G F R^{22}$ or markers for late neuronal progenitors (e.g. PAX6 and ASCL1). Both clusters also expressed markers for the oligodendrocyte lineage including the oligodendrocyte progenitor cell (OPC) markers SOX10 and $R G C C^{34}$. The cluster Neuronal was negative for all the above markers, and instead, expressed SOX6 and neuronal markers (e.g. MAP2, RBFOX1, NRXN1, and CTNNA2) (Fig. 1b, c and Supplementary Fig. 2). SOX6 is a transcription factor expressed in early OPCs, but has also been associated with the development of interneurons ${ }^{35-37}$. The final cluster that we identified only expressed LYZ and SPINT2 as cluster marker genes (Supplementary Fig. 2 and Supplementary Data 2). These two clusters only contained CD271-CD11b-GLT1- $1^{-}$sorted cells.

To further substantiate the identity of the two Progenitor and the Neuronal clusters we performed Gene ontology (GO) analysis on all highly expressed genes (adj $P$-value $<0.01$ ) (Supplementary Data 2) within the clusters: Progenitors 1, Progenitors 2, and Neuronal. Cluster Progenitors 1 and 2 showed enrichment for GO terms related to central nervous system development, axonogenesis, and glial cell development (Supplementary Fig. 3). Cluster Neuronal showed enrichment for terms related to protein modification, cell adhesion, and glutamate receptor binding. These GO analyses corroborate the identities of the three clusters as progenitors and neuronal.

Progenitors isolated from the aged human SVZ are OPCs. As the gene signature of aged human SVZ progenitors suggested an OPC identity we compared our data to the dataset from Zhong et al. ${ }^{38}$ and Jäkel et al. ${ }^{39}$. Zhong et al. ${ }^{38}$ isolated cells from the fetal human brain at different gestational stages and Jäkel et al. ${ }^{39}$ isolated white matter cells from healthy donors aged between 35 and 82 years (mean age 60 years). We used Seurat v3.2.2 to run an integrative analysis on the three datasets. This revealed several clusters including early progenitors, late progenitors, and migrating neurons (Fig. 2a-c). Moreover, we observed substantial mixing of cells from the different datasets, arguing against clustering due to batch effects. Neuronal lineage clustered together, and include mostly fetal cells and mid-aged cells from Jäkel et $\mathrm{al}^{39}$. Microglia from our dataset clustered with microglia from the two other studies. Cells from the OPC lineage from our dataset formed clusters with OPC lineage cells from Jäkel et al. ${ }^{39}$, and fetal OPCs (Fig. 2a, b and Supplementary Data 3). We next performed clustering analysis on cells from the OPC lineage only, which revealed seven subclusters (Fig. 2d, e). These subclusters corresponded to early OPCs (PDGFRA and SOX6), late OPCs 

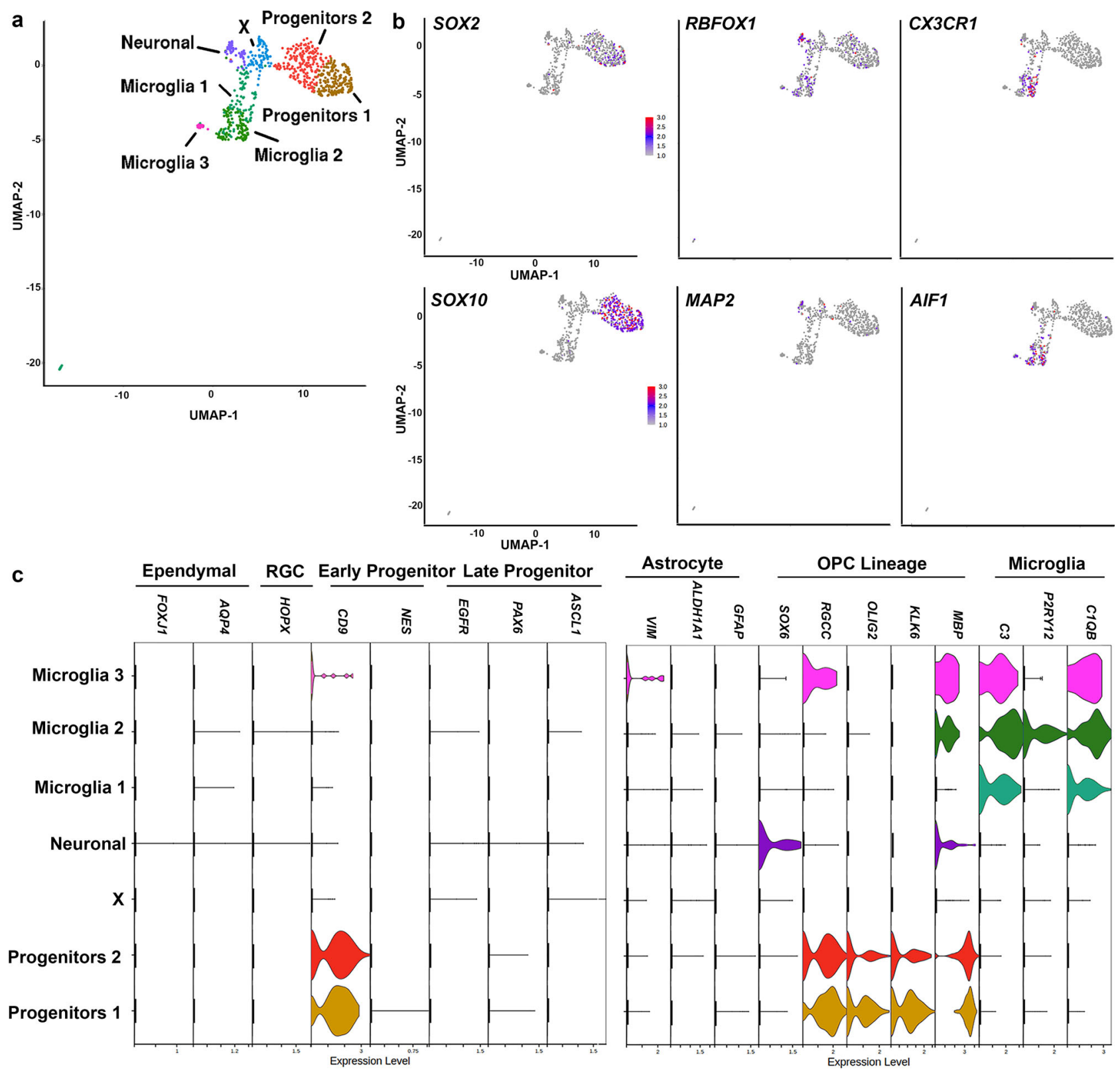

Fig. 1 Characterization of the aged human SVZ at the transcriptome level. a UMAP plot identifies seven clusters of cells, corresponding to two progenitor clusters, one neuronal, and three microglia clusters. b Feature plots showing the expression of canonical markers for the clusters: Progenitor 1 and 2 (SOX2; SOX10), Neuronal (RBFOX1; MAP2), and Microglia 1, 2, and 3 (CX3CR1; AIF1). c Feature plots showing the absence or presence of markers for ependymal, radial glial cell (RGC), early progenitor, late progenitor, astrocytes, oligodendrocyte progenitor cell (OPC) lineage and microglia. ( $n=3$ donors; 2 females, aged 95 ( 504 cells) and 96 years ( 118 cells); 1 male, aged 72 years (116 cells)).

(SOX10 and SOX2), and oligodendrocytes (KLK6 and OPALIN) (Fig. 2f). Our analysis showed that from the 395 progenitor cells that we analyzed, 138 cells corresponded to late OPCs and the remaining cells to oligodendrocytes (Supplementary Data 3). We performed SOX10 immunofluorescence staining on post-mortem human brain tissue, which showed that only a few SOX2 progenitors in the SVZ are SOX10 positive (Supplementary Fig. 4).

Cell-cycle inhibitors increase in OPCs from the aged SVZ. Analysis of the expression of a panel of markers for the oligodendroglial cell lineage further confirmed the OPC identity of the $\mathrm{CD} 271^{+}$progenitor cells (Fig. 3a). We next identified genes that were differentially expressed over time using Monocle3 v0.2.3.0 (Supplementary Data 4). One of the genes that was differentially expressed over time was SFRP1, which increased in expression with age (Supplementary Data 4). SFRP1 is an antagonist of the Wnt pathway, thereby inhibiting cell proliferation ${ }^{40,41}$. This is interesting as the mechanisms that regulate quiescence of progenitors from the human SVZ are unclear. Therefore, we compared the expression of several proliferation and cell cycle markers in fetal, mid-aged, and aged OPC lineage cells. As expected, markers for proliferation and cell cycle progression were mostly absent in the mid-aged and aged OPC lineage cells (Fig. 3b), while markers for quiescence and cell cycle arrest were highly expressed in mid-aged and aged OPC lineage cells, in particular CDKN1B (i.e. P27), CDKN1C (i.e. P57), and SFRP1 (Fig. 3c).

Cell-cycle inhibitors are expressed in the aged human SVZ. P57 is a known marker for stem cell quiescence in rodents ${ }^{9,23}$ and 
a

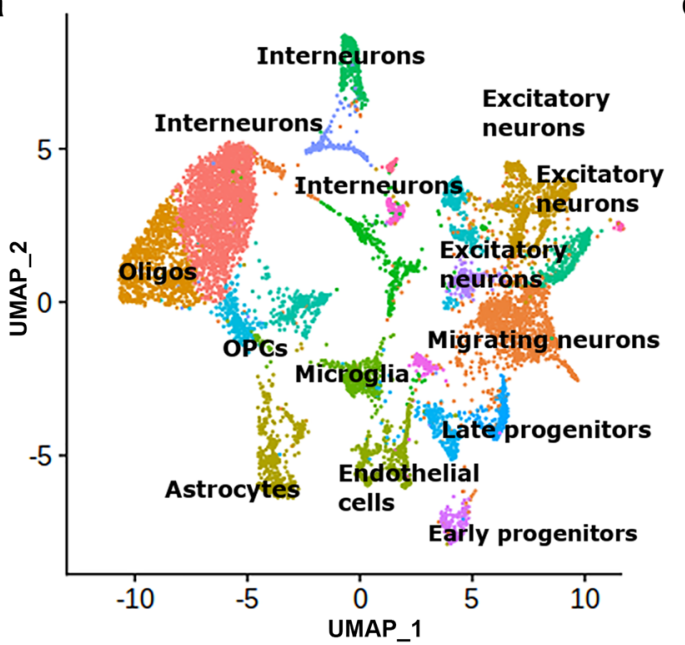

b
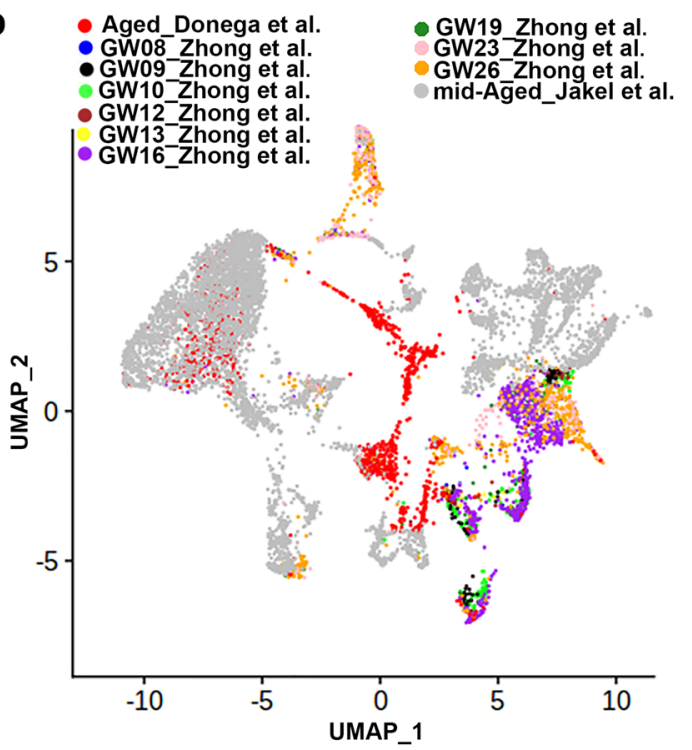

f
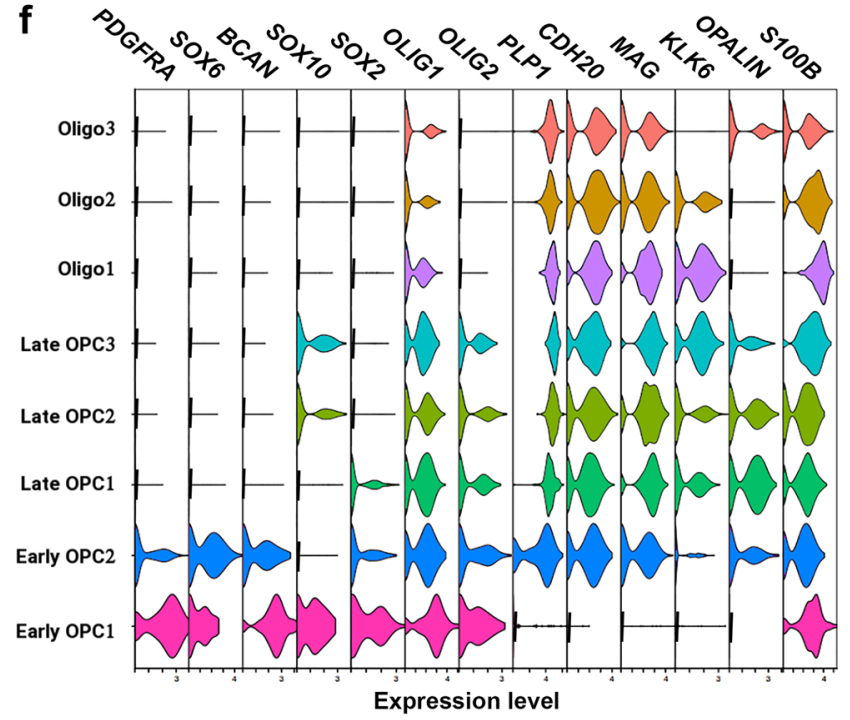
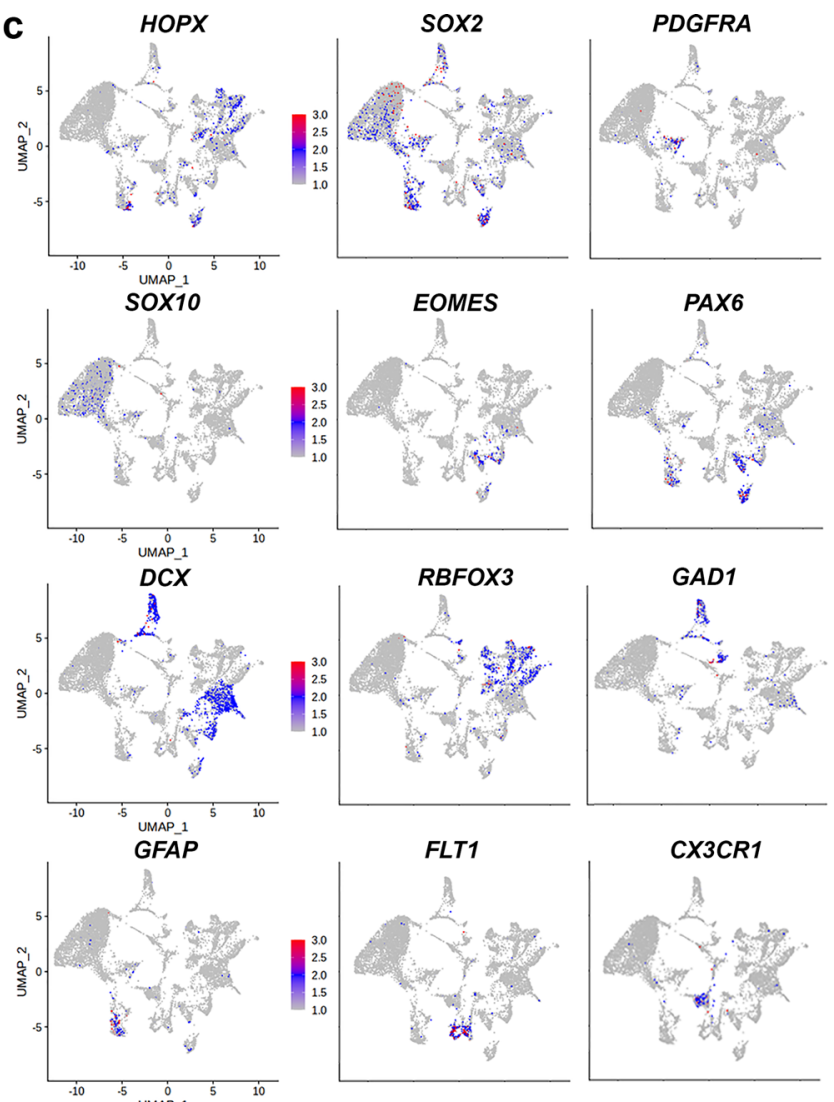

d Aged_Donega et al. GW23_zhong et al.

- GW12 Zhong et al. GW26 Zhong et al.

- GW16_Zhong et al. mid-Ağed_Jakel et al. - GW19_Zhong et al.

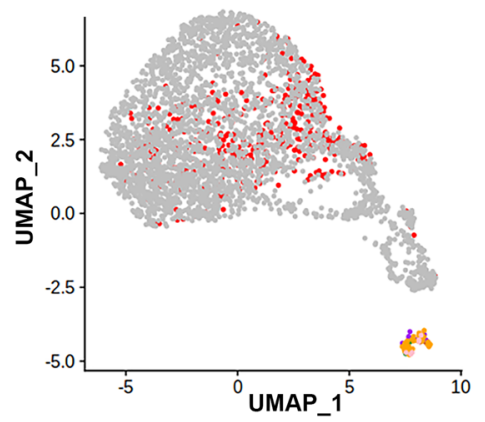

e

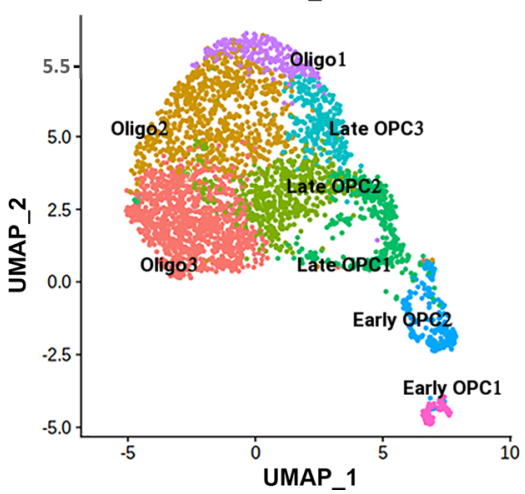

Fig. 2 CD271 ${ }^{+}$cells correspond to late OPCs and oligodendrocytes. $\mathbf{a}, \mathbf{b}$ UMAP projection of cell clusters shown by cell population (a) and origin of individual cells (b). c Feature plots for canonical markers of different cell populations. d, e UMAP projection of the origin of individual cells (d) and OPC lineage subclusters (e). $\mathbf{f}$ Violin plots of markers enriched in specific oligodendrocyte subpopulation. Gene expression level plotted as normalized counts. GW gestational weeks. ( $n=3$ donors; 2 females, aged 95 ( 504 cells) and 96 years (118 cells); 1 male, aged 72 years (116 cells)). 

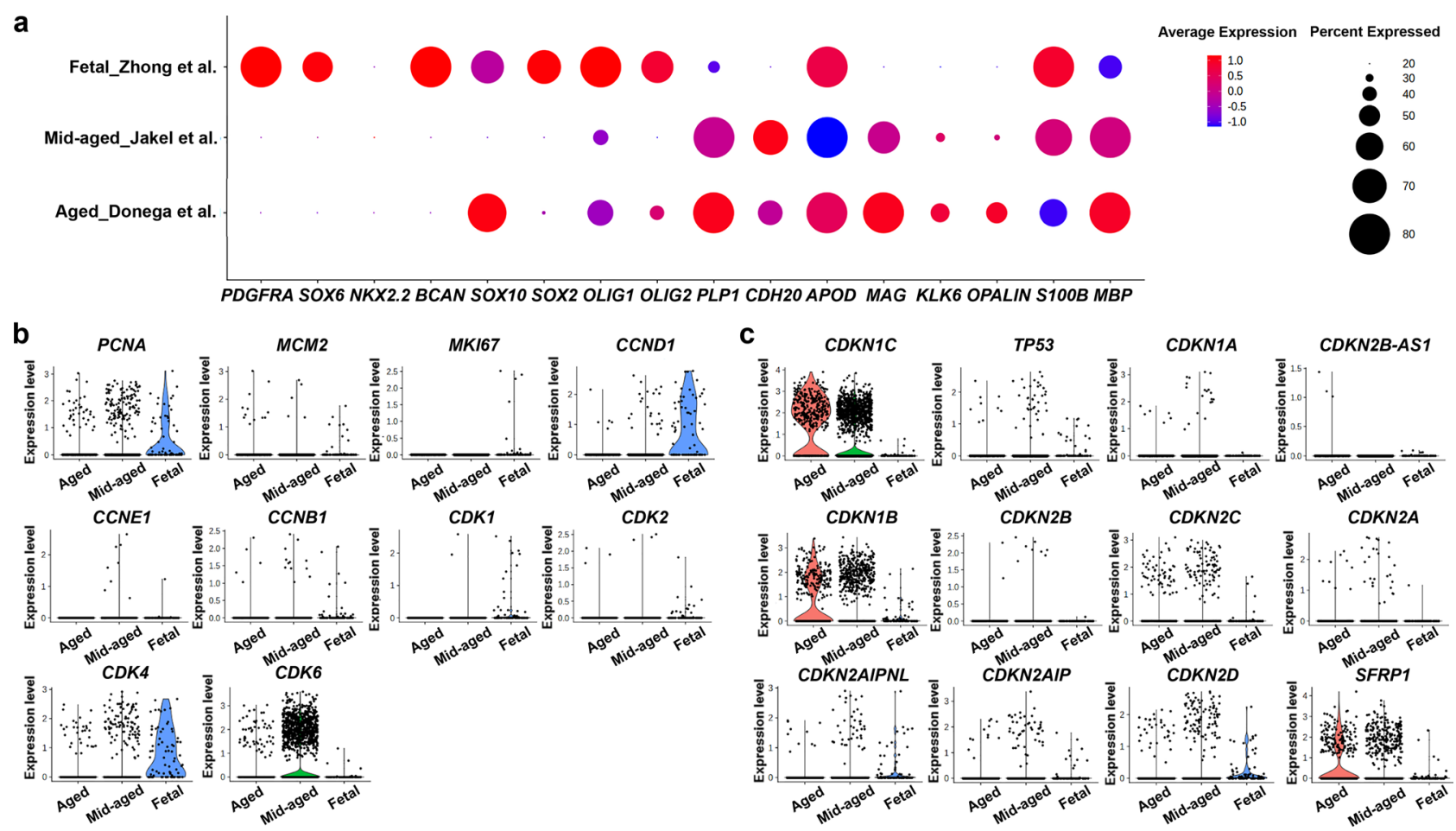

Fig. 3 Increased expression of cell cycle inhibitors in OPCs from the aged human SVZ. a Dot plot showing the expression level (red = high, blue = low) and percentage of cells expressing markers of the oligodendroglial lineage. $\mathbf{b}, \mathbf{c}$ Violin plots of markers for proliferation and cell cycle progression (b) and of markers for quiescence and cell cycle inhibition (c) in cells from the OPC lineage from Zhong et al. ${ }^{39}$, Jäkel et al. ${ }^{42}$, and present study (Donega et al.). ( $n=3$ donors; 2 females, aged 95 (504 cells) and 96 years (118 cells); 1 male, aged 72 years (116 cells)).

SFRPs are a family of biphasic regulators of Wnt signaling expressed in the nucleus or cytoplasm of the cell ${ }^{40-42}$. SFRP1 is mainly expressed in late OPCs (Fig. 4a) and is the only member of the SFRP family that is expressed in aged OPCs (Supplementary Fig. 5a). In contrast, P57 is expressed in both late OPCs as well as oligodendrocytes (Fig. 4b). To characterize the expression pattern of both P57 and SFRP1 in the aged SVZ, we performed immunofluorescence staining on post-mortem human brain tissue (Supplementary Data 5 ). In the aged SVZ around $25 \%$ of SFRP1 ${ }^{+}$cells in the SVZ expressed SOX2 (Fig. 4c, d). SFRP1 expression is not limited to progenitors, as it is also highly expressed in ependymal cells, cortical neurons (Supplementary Fig. 6a) and OLIG2 ${ }^{+}$cells in the SVZ (Supplementary Fig. 6b, c). While SFRP1 is expressed in the nucleus of progenitors (Fig. 4c) and ependymal cells, it is also expressed in the cytoplasm of neurons (Supplementary Fig. 6a).

SFRP1 inhibits the Wnt pathway by binding to Wnt ligands and by directly binding to $\beta$-catenin in the nucleus ${ }^{42}$. To determine whether SFRP1 expression correlated with a quiescent state, we assessed the expression of P57 in SFRP1 $1^{+}$cells in the SVZ only. Our results showed that around $78 \%$ of the SFRP1 ${ }^{+}$ cells in the SVZ expressed P57 (Fig. 4e, f). The majority of P57 ${ }^{+}$ cells were positive for SFRP1 $(77.79 \pm 14.89)$. This suggests that in the adult SVZ, SFRP1 is mostly expressed by quiescent/primedquiescent stem cells. We confirmed that SFRP1 is also expressed by post-mitotic progenitors of the fetal human brain at nine gestational weeks (Fig. 4g). Immunofluorescence staining of SFRP1 expression in the SVZ from aged, mid-aged, and fetal post-mortem brain shows an increase in the number of SFRP1 ${ }^{+}$ cells from mid-aged (mean age of 61 years) to aged (mean age of 91 years) (Fig. 4h) and from GW9 to GW16-17 (Fig. 4i). We also confirmed in our bulk RNAseq dataset ${ }^{31}$ that SFRP1 has the highest expression from the SFRP family members, in both $\mathrm{CD} 271^{+}$cells and SVZ homogenate isolated from post-mortem brain tissue from healthy donors (Supplementary Fig. 5b).
SFRP1 inhibition promotes proliferation in iPSC-derived NSC. A previous study showed that proliferation and differentiation increases during early corticogenesis in Sfrp $1^{-1-}$ mouse embryos ${ }^{40}$. Therefore, we assessed the effect of inhibiting SFRP1 function on proliferation of human NSCs by using a human iPSC-derived neural stem cell line to model human NSCs in vitro. This was done with the small molecule WAY-316606, which is known to sequester SFRP1 in vitro. This molecule prevents SFRP1 from binding to Wnt ligands, thereby stimulating the Wnt pathway ${ }^{43}$. We first confirmed the expression of SFRP1 protein in human iPSC-derived NSCs (Fig. 5a). Most cells expressed SFRP1 protein in the cytoplasm and nucleus, while in some cells cytoplasmic expression was absent. Sequestration of SFRP1, stimulated proliferation of iPSC-derived NSCs $72 \mathrm{~h}$ after stimulation in vitro (Fig. 5b-e). This effect is dosagedependent (Supplementary Figure 7). Stimulation with WAY increased the number of cells by two fold (Fig. 5b). While we observed an increase in SOX2 ${ }^{+}$cells, the percentage of $\mathrm{KI} 67^{+}$iPSCderived NSCs did not increase when compared to control condition (Fig. 5d-f). Our data therefore, suggest that this increase in cell number is mediated by a shortening of the cell cycle rather than an increase in cell activation. This can be explained by the fact that iPSC-derived NSCs do not exit the cell cycle, and instead remain actively cycling. To confirm that the observed effect is mediated by increased activity of the canonical Wnt pathway we performed a Topflash luciferase reporter assay on HEK293 cells. Our results confirmed that the small molecule WAY-316606 activates the canonical WNT pathway through inhibition of SFRP1 (Supplementary Fig. 8). WAY-316606 acts specifically on SFRP1 and does not activate the Wnt pathway when in presence of SFRP5, an SFRP isoform that promotes NSC quiescence in the mouse SVZ?.

SFRP1 is expressed in the postnatal mouse brain. To determine whether SFRP1 inhibition also stimulates proliferation of progenitors in vivo, we first assessed the expression pattern of SFRP1 
a

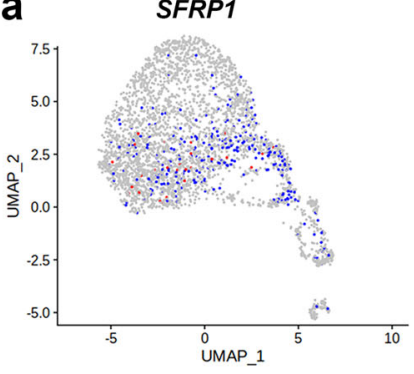

b

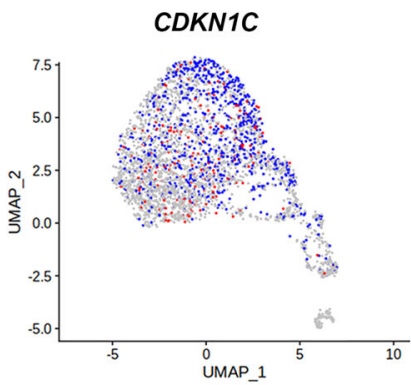

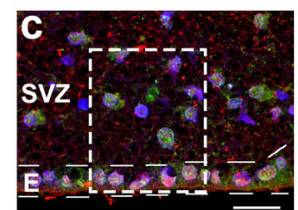

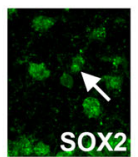

d

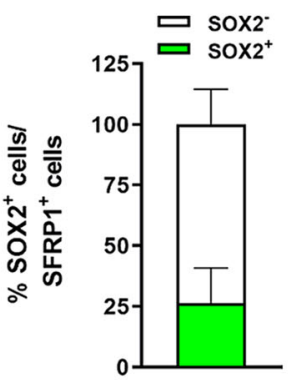

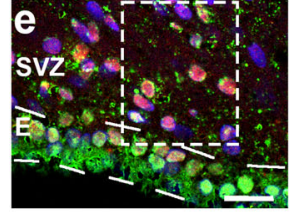

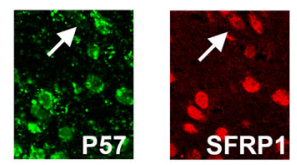

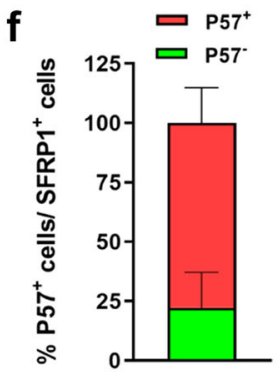
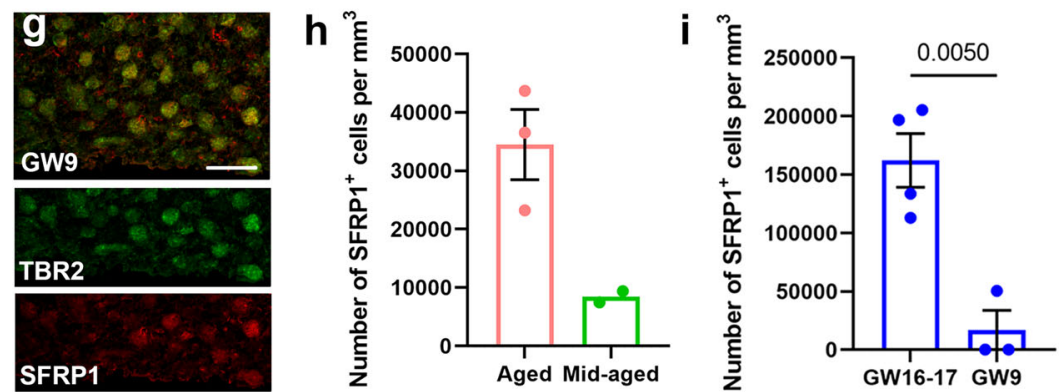

Fig. 4 Cell cycle inhibitors are expressed in the aged human SVZ. a, b Feature plot showing the expression of CDKN1C (P57) (a) and SFRP1 (b) ( $n=3$ donors; 2 females, aged 95 (504 cells) and 96 years (118 cells); 1 male, aged 72 years (116 cells)). c Representative image of SFRP1+ progenitors in the aged human SVZ. $\mathbf{d}$ Quantification of the percentage of SFRP1 ${ }^{+}$cells that express the stem cell marker SOX2 $(n=3$ donors). e Representative image of SFRP1 ${ }^{+}$and P57 ${ }^{+}$cells in the aged human SVZ. $\mathbf{f}$ Quantification of the proportion of SFRP1+ cells that express P57 ( $n=3$ donors). $\mathbf{g}$ Representative images of SFRP1 ${ }^{+}$cells in the germinal region of the fetal human brain at GW9 co-expressing TBR2. $\mathbf{h}$ Quantification of SFRP1 ${ }^{+}$cells in the SVZ of aged $(n=3$ donors; 2 females aged 92 and 99 years, 1 male aged 82 years) and mid-aged ( $n=2$ donors; 2 females aged 62 and 50 years) post-mortem brain tissue. i Quantification of SFRP1+ cells in the SVZ of GW9 ( $n=3$ donors; 2 females, 1 male) and GW16-17 ( $n=4$ donors; 2 females, 2 males) post-mortem brain tissue. Data represented as mean \pm SEM when $n>2$. Data represented as mean when $n=2$. Two-tailed Unpaired Student's $t$ test. Hoechst was used as a nuclear counterstaining. E ependymal layer, GW gestational weeks. Scale bar $=20 \mu \mathrm{m}$. Source data are provided as a Source Data file.

over time in the embryonic and postnatal mouse brain. In situ hybridization (ISH) data from Allen Brain Atlas showed a gradual increase in SFRP1 expression from E11.5 to E18.5 (Fig. 6a). During the embryonic period, SFRP1 is mainly expressed in the germinal regions. Following birth, SFRP1 expression decreases in the SVZ, while increasing in regions outside the SVZ. We confirmed the ISH data by performing immunofluorescence staining for SFRP1 on P1 and P67 mouse brains. This showed expression of SFRP1 in the SVZ, striatum, and cortex in P1 mouse brain (Fig. 6b-d), and a strong decrease in SFRP1 expression in the SVZ in P67 mouse brains (Fig. 6e-g). Kalamakis et al. ${ }^{7}$ showed that from all members of the SFRP family, only SFRP5 expression increased with time in the mouse SVZ, while SFRP1 expression decreased (Supplementary Fig. 5c). Thus, in contrast to the expression pattern of SFRP1 in the human SVZ, its expression is highest in the early postnatal mouse SVZ.

Inhibition of SFRP1 stimulates the Wnt and Notch pathways. Previous studies showed that SFRPs are multifunctional proteins that regulate both Wnt and Notch signaling ${ }^{40,42}$, through which they regulate dopamine neuron development ${ }^{44}$ and cortical expansion ${ }^{40}$. We first assessed whether inhibiting SFRP1 function increased activation of the Wnt and Notch pathways in vivo. SFRP1 was prevented from binding to Wnt ligands by the administration of the small molecule WAY-316606 to two days old mouse pups. We assessed this in the early postnatal mouse brain, as SFRP1 levels are highest in the SVZ at this age (Fig. 6 and Supplementary Fig. 5c). The entire SVZ was dissected $72 \mathrm{~h}$ after treatment with WAY-316606 for RT-PCR analysis, focusing on Wnt and Notch pathway-related genes. Our results show a 3.5-fold increase in Cyclin d1 (Ccnd1) $(P=0.0079)$, which promotes cell proliferation ${ }^{45}$ (Fig. $\left.7 \mathrm{a}\right)$. p57 $(C d k n 1 c)$ expression did not change $(P=0.4812)$. Moreover, some key genes of the Wnt signaling (Fzd7 $P=0.0025$, Ctnnb1 $P=0.0203$, and Lef1 $P=0.0497)$ and the Notch signaling (Hes5 $P=0.0131$, and Nrarp $P=0.0041)$ were also increased following administration of the small molecule WAY-316606 (Fig. 7a). Administration of WAY also enhanced the expression of $D c x(P=0.0007)$ and CNPase $(P=0.0466)$ genes, suggesting increased specification towards neuronal and OPC lineages (Fig. 7b).

SFRP1 inhibition increases activation of SVZ progenitors. We next determined whether WAY-316606 administration would stimulate progenitor proliferation also in vivo. To determine if inhibiting the function of SFRP1 increases the number of $\mathrm{GFP}^{+}$ cells and their migration away from the SVZ, we specifically labeled progenitors from the dSVZ by dorsal electroporation of a GFP plasmid at P2 and terminated the pups $72 \mathrm{~h}$ after administration of WAY-316606. Our results show a 1.6-fold increase in the number of $\mathrm{GFP}^{+}$cells in the dSVZ (Fig. 8a, b). We did not see a significant increase in migration towards the cortex. The increase in the number of $\mathrm{GFP}^{+}$cells in the $\mathrm{dSVZ}$ correlated with a 2-fold increase in proliferating cells in the dSVZ (Fig. 8c). There was also a 1.6 -fold increase in $\mathrm{Ki}^{+} 7^{+}$cells in the $1 S V Z$ (Fig. 8d). Our results show that while the number of Sox $2^{+}$progenitors remains constant in the $1 S V Z$, and increases with 2 -fold in the dSVZ, there was a 3 -fold decrease in the mSVZ after administration of WAY-316606 (Fig. 8e, f). There was a significant increase in the number of Olig2 ${ }^{+}$cells in both the dorsal and lateral SVZ (Fig. 8g, h).

\section{Discussion}

Although NSCs are present in the adult human SVZ, few neurons are generated after birth ${ }^{2,4}$. NSCs of the rodent SVZ become increasingly quiescent during aging. Studies in rodents suggest that NSC quiescence is regulated by both intrinsic and extrinsic 
a
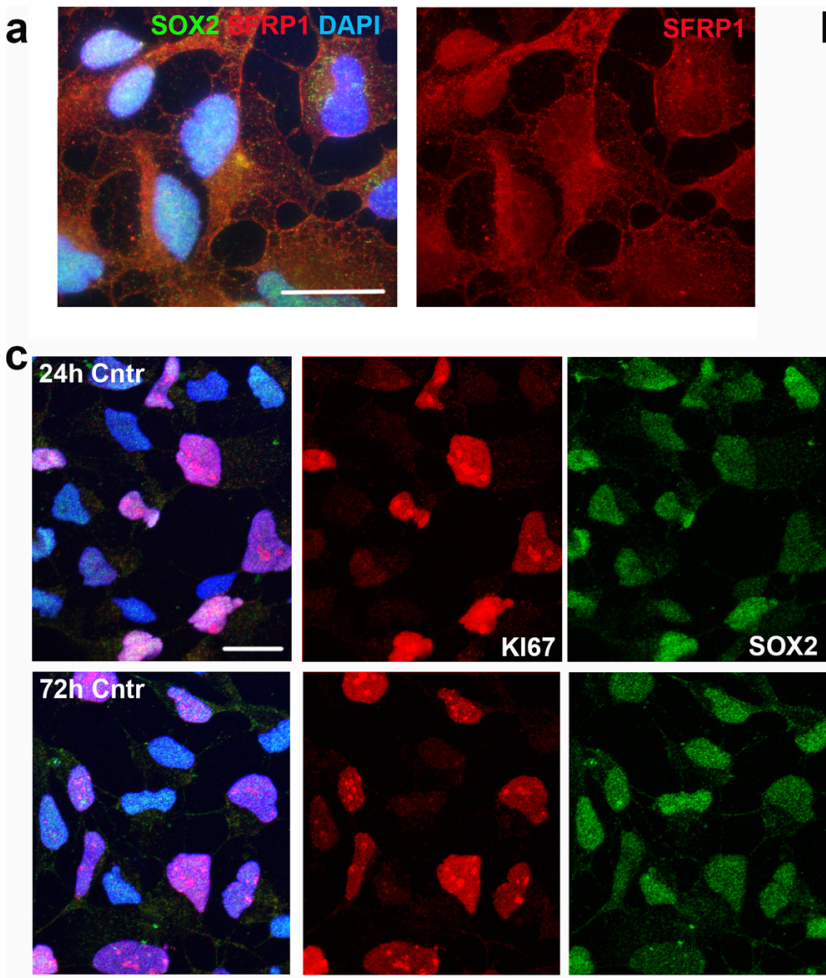

d
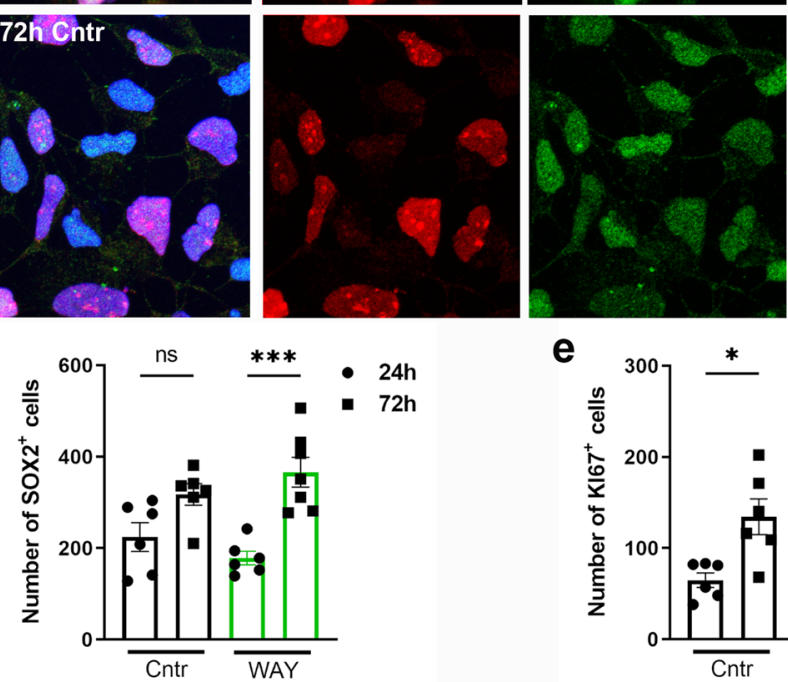

e b

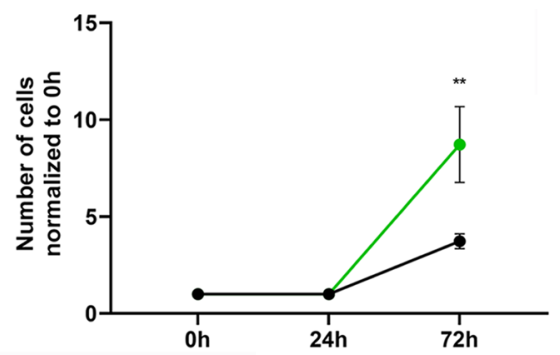

- Control

- WAY (2UM)
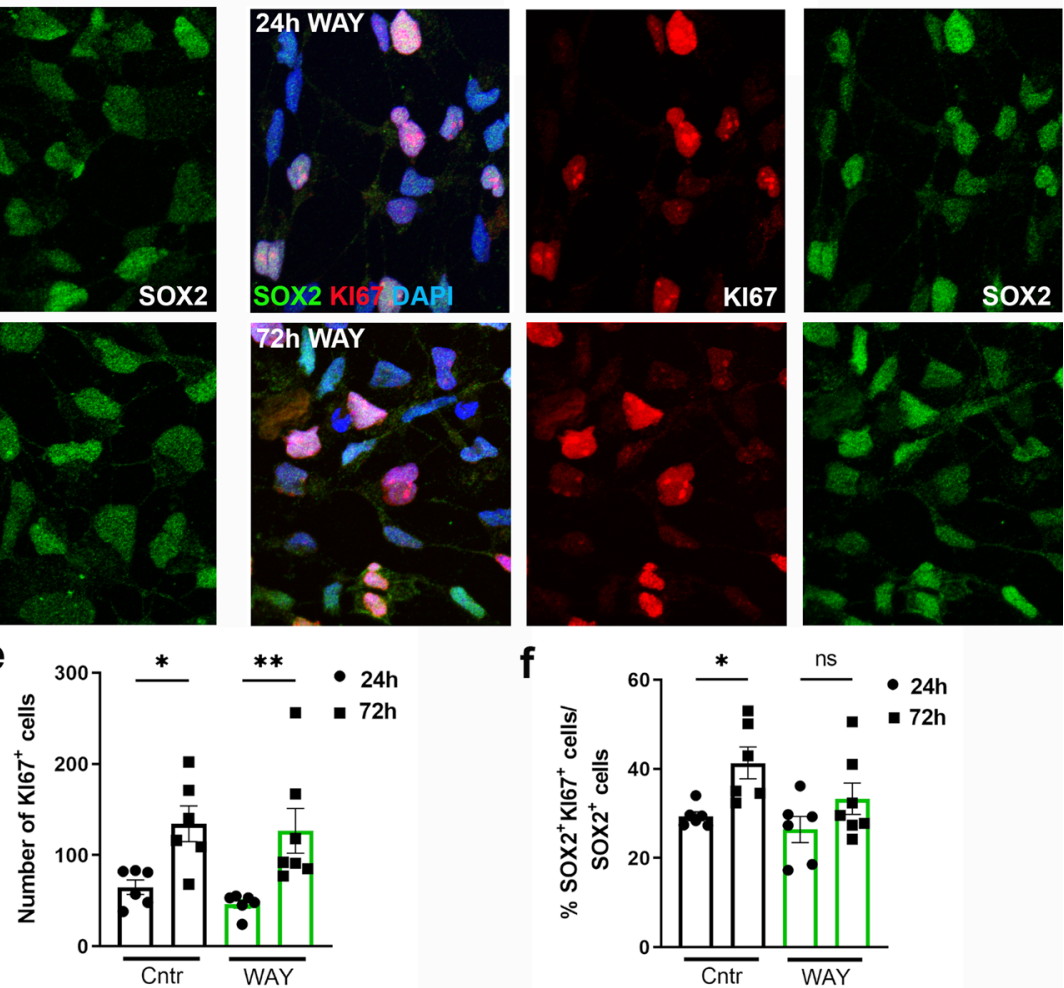

Fig. 5 Stimulation of proliferation in iPSC-derived NSCs by inhibition of SFRP1. a Representative image of SFRP1+ iPSC-derived NSCs showing both nuclear and cytoplasmic labeling. b Quantification of the number of cells in control and stimulated condition over time ( $n=3$ biological replicates),

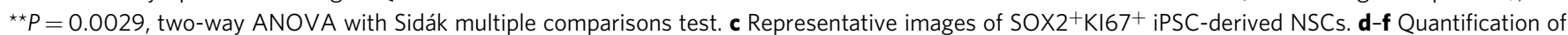
the number of SOX2+ cells $\left(n=6\right.$ images per condition), ns $=0.0529,{ }^{\star \star \star} P=0.0001(\mathbf{d}),{ }^{*} \mathrm{KI67}+{ }^{\star}{ }^{\star} P=0.0218,{ }^{\star}{ }^{\star} P=0.0061(\mathbf{e})$ and, percentage of proliferating SOX2 ${ }^{+}$cells, ${ }^{\star} P=0.0358$, ns $=0.3093$ (f). Data presented as mean \pm SEM. One-way ANOVA with Sidák multiple comparisons test. ns not significant, Cntr control, h hour. Scale bar $=20 \mu \mathrm{m}$. Source data are provided as a Source Data file.

factors (e.g. inflammatory signaling in the SVZ) $7,8,14-23$. The molecular mechanisms that maintain progenitors of the adult human brain quiescent are still unclear. Here, we identify SFRP1, an inhibitor of the canonical Wnt pathway, as a potential target to stimulate progenitor proliferation and differentiation in the adult human SVZ. We show both in vitro, in a human iPSC-derived NSC line, and in vivo, in mice, that inhibiting SFRP1 function with the administration of WAY-316606 increases activation of progenitors, likely by stimulating the activity of both Wnt and Notch pathways. Our work identifies the Wnt antagonist SFRP1 as a potential signal that maintains quiescence of progenitors of the aged human SVZ.

To characterize progenitors from the aging human SVZ, we obtained tissue from donors without neurological or psychiatric disease, which was confirmed by a thorough pathological assessment. This is important to avoid confounding factors due to changes in the neurogenic niche environment because of diseaserelated mechanisms. As expected, the assessment also showed amyloid $\beta$ deposition and neurofibrillary degeneration to varying degrees in the donors. Although we show that there is no segregation of cells by donor, the differences in degree of pathology and in age, should be kept in mind and might have influenced the number of progenitors that could be isolated, as well as their gene signature.

Interestingly, we show that progenitors from the adult human SVZ are primed towards the oligodendroglial lineage, as genes from this lineage are highly expressed, while canonical markers of the neuronal lineage are practically absent. Integration of our data with published datasets from the fetal forebrain ${ }^{38}$ and adult white matter ${ }^{39}$, revealed the CD271 ${ }^{+}$cells to be late OPCs. We cannot exclude that CD271 may label a subpopulation of progenitors in the human SVZ. It is likely that human progenitors of the SVZ are heterogeneous as in the rodent SVZ, where progenitors differ in their lineage specificity depending on their location within the $\mathrm{SVZ}^{46,47}$. A previous study suggested that CD271 is expressed specifically in OPCs following demyelinating brain injuries in both humans and rodents ${ }^{48}$. Therefore, we cannot conclude, based on our current results, that either early OPCs or NSCs are absent from the aged human SVZ.

The turnover rate of oligodendrocytes stabilizes around five years of age and remains low throughout the human lifespan ${ }^{49}$. Our results indicate that this low turnover rate is not caused by 

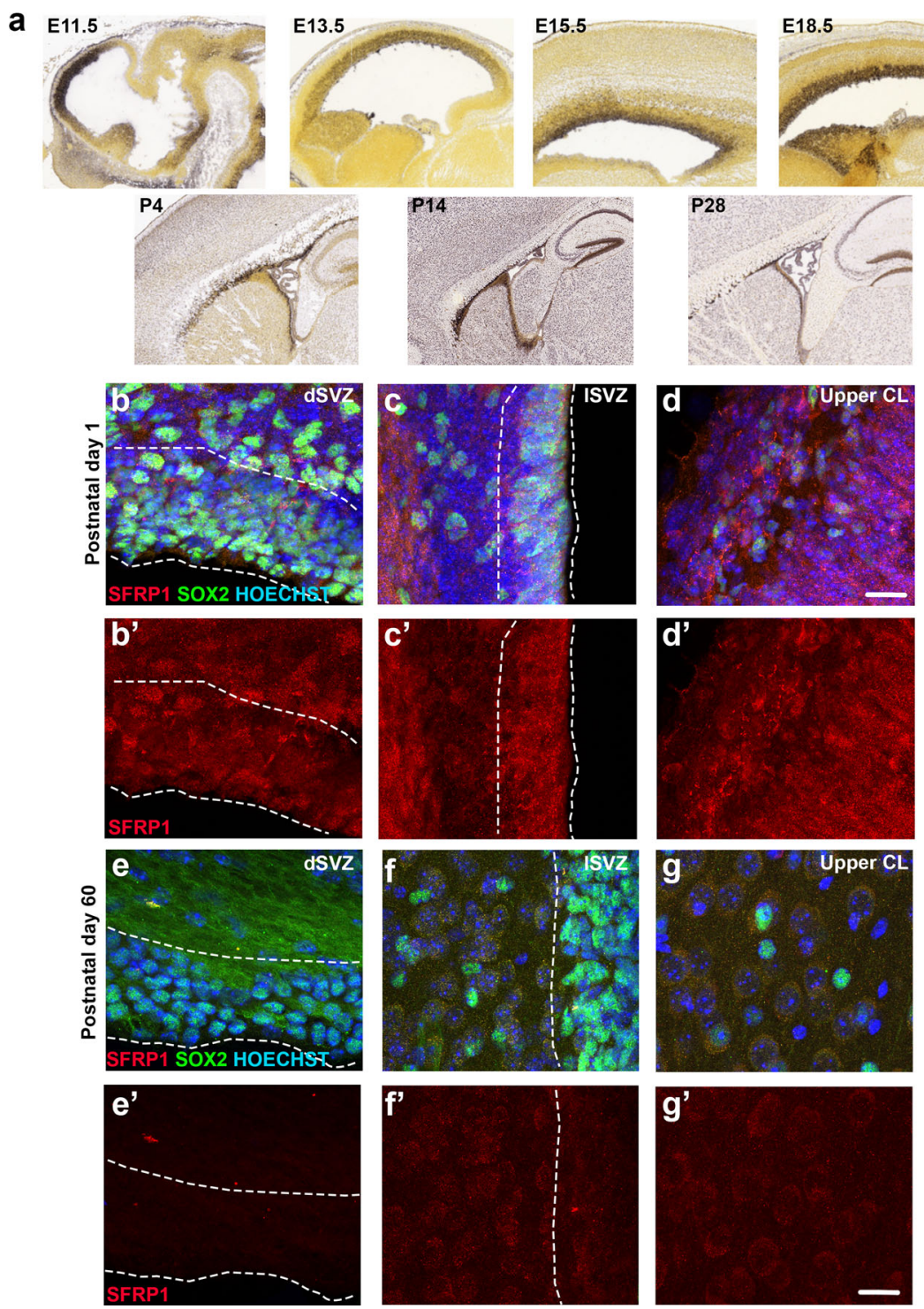

Fig. 6 SFRP1 is expressed in the neonatal and adult mouse brain. a In-situ hybridization images from the Allen Brain Atlas showing SFRP1 expression in the mouse brain at different developmental stages. b-d Representative images of SFRP1 expression in the mouse brain at postnatal day 1 ( $n=3$ mice).

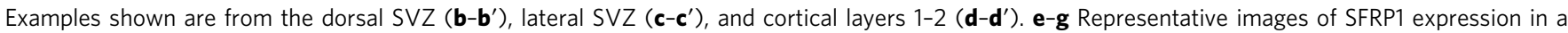
60 days old mouse brain ( $n=4$ mice; 3 females and 1 male). Examples show dorsal SVZ (e-e'), lateral SVZ (f-f') and upper cortical layer ( $\left(\mathbf{g}-\mathbf{g}^{\prime}\right)$. Hoechst was used as a nuclear counterstaining. E embryonic day, P postnatal day, dSVZ dorsal SVZ, ISVZ lateral SVZ, CL cortical layer. Scale bar $=20 \mu$ m.

the absence of OPCs in the SVZ, but rather due to increased quiescence. Here, we identified SFRP1, a Wnt pathway antagonist, as a possible signal that maintains late OPCs in a quiescent state in the human SVZ. SFRP expression is not restricted to NSCs, but is also expressed by astrocytes ${ }^{50}$ and microglia ${ }^{51}$. Two other members of the SFRP family, SFRP3 and SFRP5, were shown to regulate NSC quiescence in the mouse brain. SFRP3 maintains NSCs in a quiescent state in the dentate gyrus ${ }^{52}$ of adult mice and its deletion increases NSC activation and maturation. SFRP5 was shown to maintain NSCs of the aged mouse SVZ quiescent and when blocked by the administration of antibodies, activation of aged NSCs was increased ${ }^{7}$. Neither SFRP3 nor SFRP5 are expressed in our datasets (Supplementary Figure 5$)^{31}$, suggesting species-specific differences in expression profile. Indeed, our data show that while in humans, SFRP1 expression in the SVZ increases with age, its expression decreases in young adult mice. Hence, SFRP1 could be the human homolog of SFRP5 in regulating NSC quiescence in the aged human SVZ.
The mechanisms through which SFRPs modulate the canonical Wnt pathway to maintain cells in a quiescent state are still unclear. Growing evidence suggests that members of the SFRP family function as tumor suppressor genes, as they are lowly expressed in different types of tumors, including meduloblastoma ${ }^{53-55}$. Methylation of the promoter region of $S F R P$, results in its decreased expression, which correlates to increased malignancy ${ }^{54}$. Indeed, low levels of SFRP1 have been shown to increase proliferation in different tumor cell lines ${ }^{53}$. A recent study proposed a Wnt-independent mechanism in which nuclear SFRP1, 2, and 5 directly bind to $\beta$-catenin, thus inhibiting its transcriptional activity and the expression of cancer stem cell related genes ${ }^{42}$. We show that inhibition of SFRP1, by the administration of WAY-316606, stimulates proliferation and differentiation of NSCs both in vitro and in vivo by activation of the canonical Wnt pathway. The luciferase Topflash reporter assay also shows that WAY-316606 acts specifically on SFRP1 and does not activate the canonical Wnt pathway when in the 
a
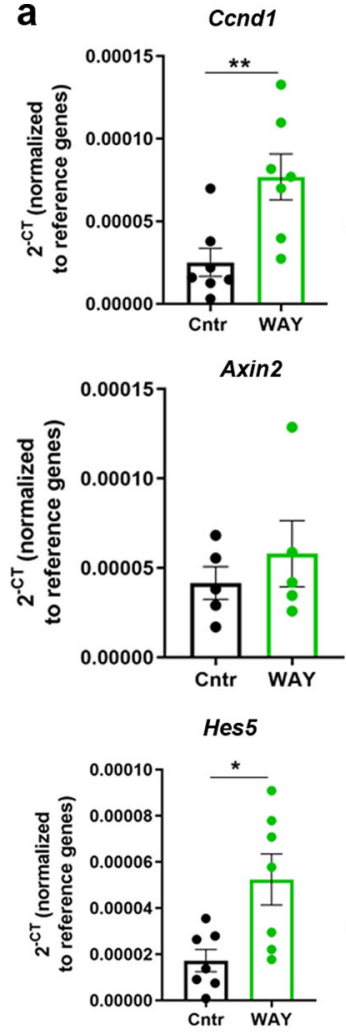

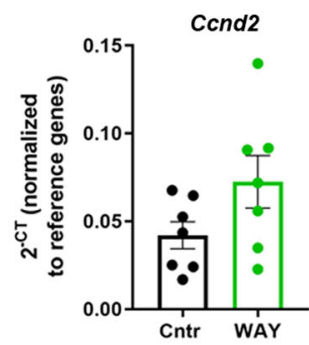

Ctnnb1
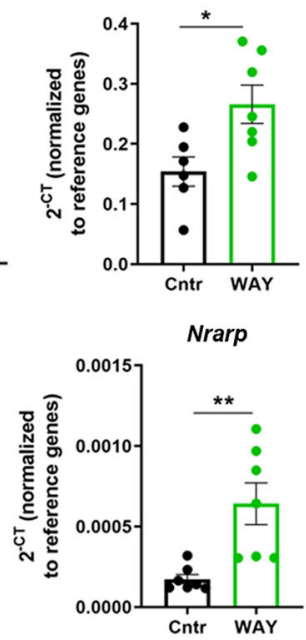

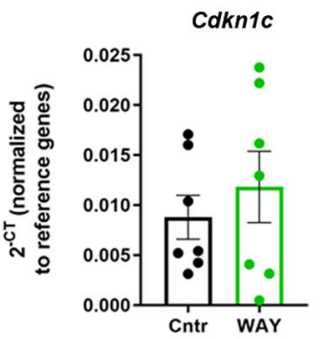

Lef1
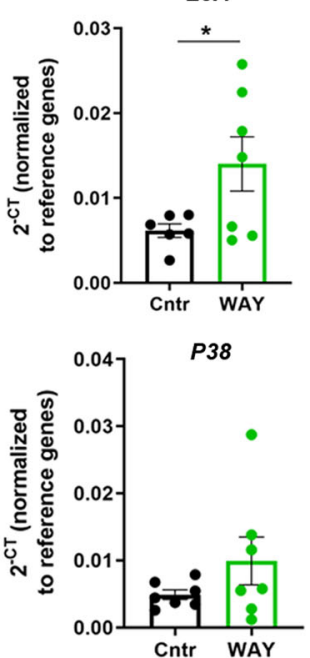
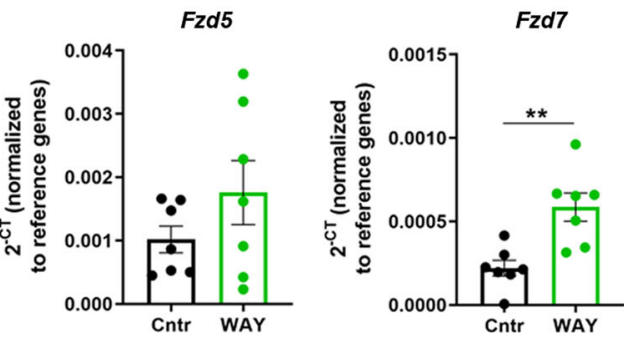

Tle1
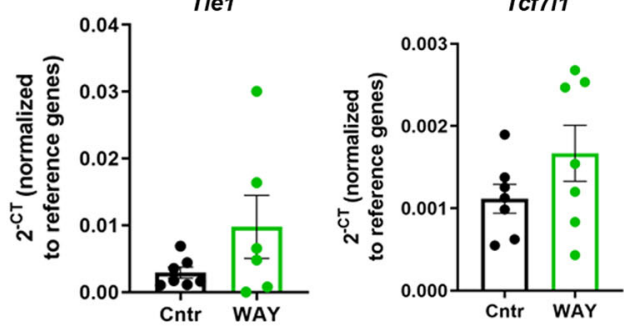

b
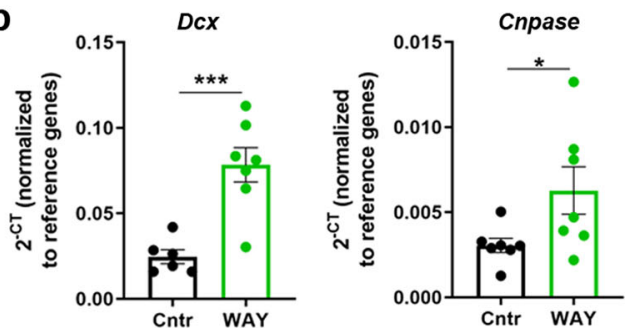

Fig. 7 Wnt and Notch pathways are activated after SFRP1 inhibition. a RT-PCR analyses of members of the Wnt and Notch signaling pathway $72 \mathrm{~h}$ after control or WAY treatment. $C$ cnd1, $P=0.0079 ; C c n d 2, P=0.0958 ; C d k n 1 c, P=0.4812 ; F z d 5, P=0.2015 ; F z d 7, P=0.0025 ; A x i n 2, P=0.4514 ; C t n n b 1$ $P=0.0203$; Lef1 $P=0.0497 ;$ Tle1, $P=0.1491 ; T c f 711, P=0.1737 ;$ Hes5 $P=0.0131 ;$ Nrarp $P=0.0041 ; P 38, P=0.1938$. b RT-PCR analyses of $D c x$ and $C N P a s e$ $72 \mathrm{~h}$ after control or WAY treatment. $D c x, P=0.0007 ; C N P a s e, P=0.0466 . n=7$ mice per group, P6; Ctnnb1, Lef1, Axin2, and Dcx had one outlier removed from control group, Grubbs test with $a=0.05$. Normalized against the levels of $\beta$-actin and Gapdh reference genes. Data presented as mean $\pm \mathrm{SEM}$. Twotailed Unpaired Student's $t$ test. ${ }^{\star} P<0.05 ;{ }^{\star \star} P<0.01 ;{ }^{\star \star \star} P<0.001$. Source data are provided as a Source Data file.

presence of SFRP5. These results are also supported by a study where bone formation was stimulated through sequestration of SFRP1 with WAY-316606 ${ }^{56}$ showing that WAY-316606 inhibits SFRP1 activity with $40 \%$, while SFRP2 and SFRP5 activities were only decreased by $2-5 \%$. All together, these data suggest that the small molecule WAY-316606 promotes the activity of the canonical Wnt pathway through inhibition of the Wnt antagonist SFRP1.

In conclusion, our work identifies SFRP1 as a potential signal that maintains progenitors of the aged human SVZ in a quiescent state, supporting the possibility to re-activate progenitors of the aged human brain to regenerate the brain following injury or neurodegenerative diseases.

\section{Methods}

Animals. All animal experiments were performed in accordance to the international guidelines from the EU directive 2012/63/EU and approved by the Experimental Animal Committee Utrecht (University Utrecht, Utrecht, Netherlands) (CCD number: AVD1150020184944). Animal experiments were carried out on wild-type C57BL/6j mice (Charles River, The Netherlands) aged 1 (P1), 2 (P2), or 60 (P60) days. Both males and females were used. The morning when a plug was observed is considered as E0.5 and the day of birth is defined as P0. Mice were housed in groups, with access to food and water ad libitum on a $12 \mathrm{~h} \mathrm{light/dark}$ cycle, humidity of $45-65 \%$ and a temperature between 20 and $24{ }^{\circ} \mathrm{C}$. Handling of pups was kept to a minimum to reduce stress to both mom and pups.

Human post-mortem brain tissue for single-cell RNAseq. Fresh human postmortem dorsal SVZ including adjoining white matter tissue ( $n=3$ donors) (Supplementary Fig. 1a) was obtained from donors without known neurological or psychiatric disease from the Netherlands Brain Bank (NBB; https:// www.brainbank.nl), Netherlands Institute for Neuroscience, Amsterdam. The NBB performs quick brain autopsies to ensure high tissue quality. All donors have given informed consent to the NBB to perform autopsies for tissue isolation, access to medical records for research purposes and consent to publish clinical information potentially identifying individuals. The independent Medical Ethics Committee of the VU University Medical Center has reviewed and agreed to the procedures of the NBB concerning donation of brain material for scientific research (2009/148) (https://www.brainbank.nl/about-us/the-nbb/). Autopsies are performed by the NBB at the designated premises of the VU Medical Center in Amsterdam, the Netherlands. The NBB adheres to the standards for quality, safety and ethics for obtaining and handling of human tissue, as described in BrainNet Europe's Code of Conduct for brain banking. This study was reviewed and authorized by NBB's scientific committee. The study design and conduct complied with all relevant regulations regarding the use of human study participants and was conducted in accordance with the criteria set by the Declaration of Helsinki. This study was performed according to the Dutch and European legal and ethical regulations. To ensure donor anonymity only an autopsy serial number, which is given by the $\mathrm{NBB}$, is disclosed. This number contains the year that the autopsy was performed and the number of the autopsy. Directly after autopsy, samples are placed in Hibernate-A medium (ThermoFisher Scientific, Landsmeer, The Netherlands, A1247501) and were kept cold until isolation. Samples had a mean post-mortem delay of $6.35 \mathrm{~h}$ (Supplementary Data 1$)$.

Human post-mortem brain tissue for immunofluorescence. Adult post-mortem dorsal SVZ tissue from donors without known neurological disease was obtained from the NBB (Supplementary Data 5) ( $n=5$ donors). All donors have given informed consent to the NBB to perform autopsies for tissue isolation, access to medical records for research purposes and consent to publish clinical information potentially identifying individuals. The independent Medical Ethics Committee of the VU University Medical Center has reviewed and agreed to the procedures of the NBB concerning donation of brain material for scientific research (2009/148) The NBB adheres to the standards for quality, safety, and ethics for obtaining and handling of human tissue, as described in BrainNet Europe's Code of Conduct for brain banking. This study was reviewed and authorized by NBB's scientific committee. The study design and conduct complied with all relevant regulations regarding the use of human study participants and was conducted in accordance with the criteria set by the Declaration of Helsinki. This study was performed according to the Dutch and European legal and ethical regulations. To ensure 

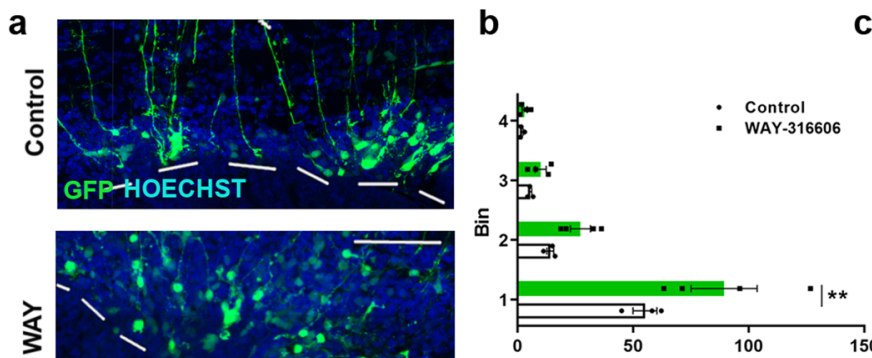

玄

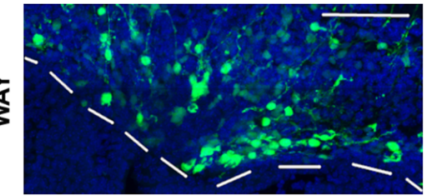

Number of $\mathrm{GFP}^{+}$cells/SVZ
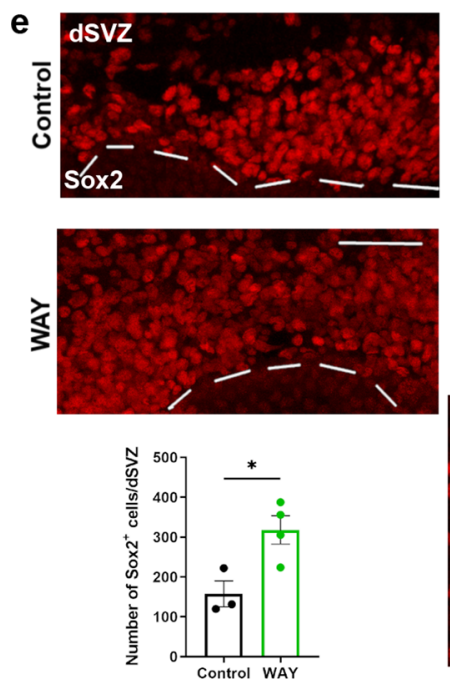
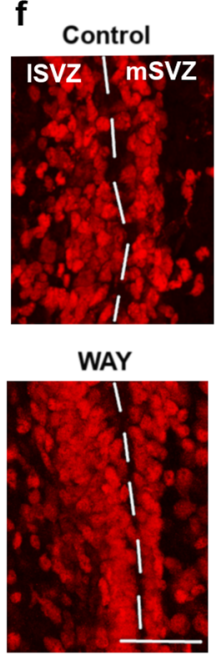

c
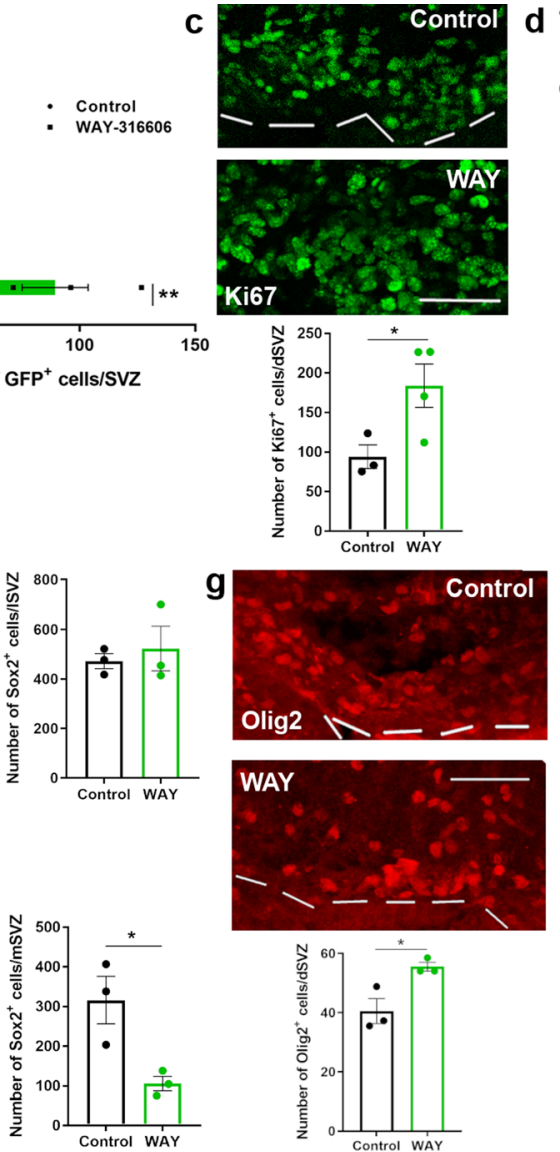
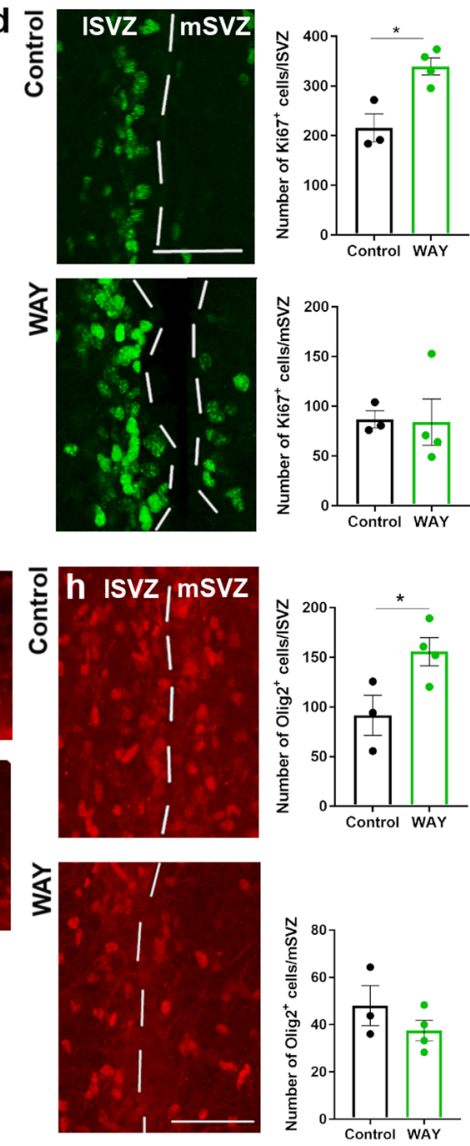

Fig. 8 Sequestration of SFRP1 increases activation of NSCs in the early postnatal mouse SVZ. a, b Representative images of GFP labeled NSCs in the dorsal SVZ (a). Increase in GFP+ cells in the dorsal SVZ following WAY administration. The region analyzed was divided into four equidistant bins, with bin one corresponding to the SVZ and bin four the area below cortical layer $6 .{ }^{\star}{ }^{\star} P=0.0056$, two-way ANOVA with Sidáks multiple comparisons test. $n=3$ mice (Control) and $n=4$ mice (WAY), P6. b. c, d Representative images and quantification of the number of Ki67+ cells in the dorsal SVZ, ${ }^{\star} P=0.0490$, $n=3$ mice (Control) and $n=4$ mice (WAY), P6. (c), lateral, ${ }^{\star} P=0.0106$, and medial SVZ, $P=0.9260, n=3$ mice (Control) and $n=4$ mice (WAY), twotailed unpaired Student's $t$ test (d) after control or WAY administration. $\mathbf{e}, \mathbf{f}$ Representative images and quantification of the number of Sox ${ }^{+}$cells in the dorsal SVZ, ${ }^{\star} P=0.0241 n=3$ mice (Control) and $n=4$ mice (WAY) (e), lateral, $P=0.6187$, and medial SVZ, ${ }^{\star} P=0.0280, n=3$ mice (Control) and $n=3$ mice (WAY), P6, two-tailed unpaired Student's $t$ test (f) after control or WAY administration. $\mathbf{g}$, $\mathbf{h}$ Representative images and quantification of the number of Olig2 ${ }^{+}$cells in the dorsal SVZ, ${ }^{\star} P=0.0280, n=3$ mice (Control) and $n=4$ mice (WAY) one outlier removed with Grubbs test with alpha $=0.05$ ( $\mathbf{g}$ ), lateral, $P^{\star}=0.0439$ and medial SVZ, $P=0.2813, n=3$ mice (Control) and $n=4$ mice (WAY), P6, two-tailed unpaired Student's $t$ test (h) after control or WAY administration. dSVZ dorsal SVZ, ISVZ lateral SVZ, mSVZ medial SVZ. Data presented as mean \pm SEM. Scale bar $=50 \mu \mathrm{m}$. Source data are provided as a Source Data file.

donor anonymity only an autopsy serial number, which is given by the NBB, is disclosed. Material was fixed in formalin and embedded in paraffin. Fetal human brain tissue was obtained from abortion material without developmental structural chromosomal abnormalities (Supplementary Data 6) from the Chinese University of Hong Kong. Forebrain tissue was obtained from gestational week (GW) $9(n=3$ donors), GW 16 ( $n=2$ donors), and GW 17 ( $n=2$ donors), fixed in 4\%-paraformaldehyde (PFA) and embedded in paraffin. Donors of fetal tissue have given informed consent to the use of the tissue for research. Women being admitted for pregnancy termination due to different clinical indications were invited to donate their conception material, including placenta, abortus and fetal blood for study. The participants understood that the fetal tissue would normally be discarded as medical waste. Participation was entirely voluntary, without compensation, and the decision of the donor did not interfere with the clinical care. Fetal tissue samples without structural and chromosomal abnormalities, were collected after the completion of the termination of the pregnancy. Involvement in the study did not confer any additional risk over the routine clinical treatment. The participants had the right to withdraw from the study and request for the collected samples to be destroyed at any time. All clinical information will remain confidential. Identity of the donor is kept anonymous by the use of a serial number. This study was performed according to the Dutch, European, and Hong Kong institutional ethical regulations for the use of abortion material. This study was approved by the Chinese University of Hong Kong - New Territories East Cluster Clinical Research Ethics Committee (CREC), Faculty of Medicine, The Chinese University of Hong Kong (under the human ethics approval reference number CREC-2004.330). The study design and conduct complied with all relevant regulations regarding the use of human study participants and was conducted in accordance with the criteria set by the Declaration of Helsinki.

Single-cell RNA sequencing. Following single-cell sorting the plate was centrifuged at $300 \times g$ for $1 \mathrm{~min}$ and kept at $-20^{\circ} \mathrm{C}$ until further processing. Sort-seq was run on single-cells as described in Muraro et al., $2016^{57}$, which is based on single-cell RNA sequencing by multiplexed linear amplification (Cel-Seq2 protocol from Hashimshony et al. ${ }^{32}$ ). Cells were lysed for $5 \mathrm{~min}$ at $65^{\circ} \mathrm{C}$, followed by dispersion of RT and second strand mixes with the Nanodrop II liquid handling platform (GC Biotech, Waddinxveen, NL). After in vitro transcription, the cDNA library was prepared according to the Cell-Seq2 protocol. The primers used consisted of 24 bp polyT stretch, a $6 \mathrm{bp}$ random molecular barcode (UMI), a cellspecific 8 bp barcode, the $5^{\prime}$ Illumina TruSeq small RNA kit adaptor (all from Illumina, San Diego, CA, USA) and a T7 promoter (ThermoFisher Scientific, Ambion AMB13345). TruSeq small RNA primers (Illumina) were used for making the Illumina sequencing libraries. Sequencing was done on the Illumina NextSeq 500 platform by sequencing paired-end at $75 \mathrm{bp}$ read length $(25 \mathrm{bp}$ from R1 and $50 \mathrm{bp}$ from R2) with a sequencing depth of $15 \mathrm{M}$ reads per 384-well plate.

Luciferase reporter (Topflash) assay. HEK293T cells (LGC Standards, Middlesex, UK, ATCC-CRL-11268) were grown in a 96 well plate and transfected $8 \mathrm{~h}$ after plating with $33 \mathrm{ng}$ Topflash (Addgene TA viral promotor, Watertown, MA, USA, 12456) and 3,3 ng Renilla (pRL-TK Renilla, Promega, Leiden, The Netherlands) using PEI transfection reagent (polyethylenimine, linear, $25000 \mathrm{mw}$, 
Polysciences 23966-2, Bergstrasse, Germany). $24 \mathrm{~h}$ after transfection, HEK293T cells were incubated with: (i) 1\% CHAPS control; (ii) recombinant human Wnt3a (Wnt3a: $180 \mathrm{ng} / \mathrm{mL}$, R\&Dsystems 5036-WN/CF); recombinant human Wnt3a with recombinant human SFRP5 protein $(5 \mu \mathrm{g} / \mathrm{mL}$, Thermo Fisher Scientific 15953709); (iii) recombinant Wnt3a with recombinant human SFRP1 protein $(5 \mu \mathrm{g} / \mathrm{mL}$, Peprotech $120-29$, London, UK); (iv) recombinant Wnt3a with recombinant SFRP1 protein and small molecule ( $2 \mu \mathrm{M}$ WAY-316606); and (v) recombinant SFRP5, recombinant Wnt3a and WAY small molecule. After $24 \mathrm{~h}$ cells were harvested and Luciferase activity was determined according to the manufacturer's manual (Dual-Luciferase Reporter Assay System, Promega, Madison, WI, USA, E1910). Values are presented as Firefly activity normalized to Renilla values and corrected for the $1 \%$ CHAPS control

Immunofluorescence staining. Immunofluorescence was performed on human post-mortem fetal and adult SVZ tissue (Supplementary Data 5 and Supplementary Data 6). $7 \mu \mathrm{m}$ thin paraffin sections were deparaffinized, washed in PBS and washed in 3\% hydrogen peroxide solution for $10 \mathrm{~min}$ to block endogenous peroxidase. Antigen retrieval was performed in $10 \mathrm{mM}$ citrate buffer $\mathrm{pH} 6.0$ at $80^{\circ} \mathrm{C}$ for $20 \mathrm{~min}$. After cooling down sections were thoroughly washed in $0.25 \%$ TritonX100 in PBS, and incubated with blocking solution 2\% BSA with $0.4 \%$ Triton-X100 in PBS at room temperature for $1 \mathrm{~h}$. Sections were incubated with primary antibodies at $4{ }^{\circ} \mathrm{C}$ overnight. The following primary antibodies were used: rabbit antiSOX2 (1:100; EMDMillipore, Burlington, MA, USA, NBP1-20136, lot Q2922255), goat anti-SOX10 (1:200; R\&Dsystems, Minneapollis, MN, USA, AF2864), goat anti-SFRP1 (1:100; R\&Dsystems, AF1384, lot IRQ061610A), mouse anti-PCNA (1:100; Santa Cruz, Dallas, TX, USA, sc-56), rabbit anti-P57 (1:100; Abcam, Burlingame, CA, USA, ab75974) and rabbit anti-OLIG2 (1:100; IBL, Minneapolis, MN, USA, 18-953, lot 1B-327). After washing, sections were incubated with the corresponding horseradish peroxidase antibody (1:500; Jackson ImmunoResearch, UK) for $1 \mathrm{~h}$ at room temperature, washed, followed by incubation with Tyramide Signal Amplification (Perkin Elmer, Waltham, USA, NEL704A001 and NEL701A001) Cy3 (1:50) for $10 \mathrm{~min}$, or with Fluorescein (1:300) for $5 \mathrm{~min}$. Sections were washed and incubated with TrueBlack for $30 \mathrm{sec}$ (Biotium, CA, USA, 23007) to quench autofluorescence and immediately washed in PBS. Nuclear counterstaining was done with Hoechst 33258 (1:5000; Invitrogen, 94403) and sections were mounted with FluorSave (EMD Millipore, 345789).

Mice were sacrificed with an overdose of pentobarbital and fixed by transcardial perfusion with PBS followed by $4 \%$ PFA (wt/vol). Brains were dissected and postfixed in $4 \%$ PFA at $4{ }^{\circ} \mathrm{C}$. Free floating vibratome serial sections were cut at a thickness of $40 \mu \mathrm{m}$. Sections were blocked in $2 \%$ BSA with $0.4 \%$ Triton-X100 in PBS at room temperature for two hours and incubated with primary antibodies diluted in $2 \%$ BSA with $0.25 \%$ Triton-X100 in PBS at $4{ }^{\circ} \mathrm{C}$ overnight. The following primary antibodies were used: rabbit anti-Sox2 (1:500; EMDMillipore), rabbit antiSfrp1 (1:500; Abcam, ab4193), mouse anti-Ki-67 (1:1000; Abcam, ab15580), and rabbit anti-Olig2 (1:200; IBL). After thorough washing, sections were incubated with the corresponding secondary antibodies conjugated to Alexa-555 or Alexa-647 (1:1000; Invitrogen) at room temperature for two hours. Nuclear counterstaining was done with Hoechst 33258 (1:5000; Invitrogen) and sections were mounted with FluorSave (EMD Millipore)

qRT-PCR. Following RNA extraction with Trizol and chloroform, RNA was precipitated in isopropanol. Reverse transcription of mRNA was done using the Quantitect Reverse Transcription kit (Qiagen GmbH, Hilden, Germany, 205311) according to the manufacturers' protocol. Primers were designed using the PrimerBLAST designing tool from the NCBI website (https://www.ncbi.nlm.nih.gov/tools/ primer-blast/; listed in Supplementary Data 7) and from Furutachi et al. ${ }^{9}$, Kase et al. ${ }^{58}$, Esteve et al. ${ }^{40}$, and Bonnefont et al. ${ }^{59}$. qRT-PCR was performed on the Quantstudio 6 Flex (Applied Biosystems, Life Technologies) using FastStart Universal SYBR green master (Rox) reagent (Roche Diagnostics GmbH, 04913914001) and analyzed using the Quantstudio Realtime PCR software (version v1.1; Applied Biosystems). Results are shown as $2^{-\mathrm{Ct}}$ values normalized to the reference genes Gapdh and $\beta$-actin.

Statistical analysis. Data shown in Figs. 4, 5, 7, 8 and Supplementary figs. 6-8 are expressed as mean \pm SEM. Measurements were taken from distinct samples. The number of samples analyzed is stated in the figure legend. Significance was tested on GraphPad Prism 7 (La Jolla, Ca, USA) with two-tailed unpaired t-test, one-way ANOVA with Sidák multiple comparisons test or a two-way ANOVA with Sidák multiple comparisons test. A $P$-value of $<0.05$ was considered statistically significant. Outliers were detected using the Grubbs test with $\alpha=0.05$.

Reporting summary. Further information on research design is available in the Nature Research Reporting Summary linked to this article.

\section{Data availability}

The single-cell RNA sequencing dataset generated in this study has been deposited in NCBI's Gene Expression Omnibus database under the accession number: GSE164986. The source data generated in this study are provided in the Source Data file. The Zhong et al. ${ }^{38}$ and Jäkel et al. ${ }^{39}$ data used in this study are available in the NCBI's Gene Expression Omnibus database under accession codes GSE104276 and GSE118257, respectively. Source data are provided with this paper.

\section{Materials availability}

This study did not generate new unique reagents.

\section{Code availability}

No new code was generated.

Received: 20 January 2021; Accepted: 28 January 2022; Published online: 24 February 2022

\section{References}

1. Kempermann, G. et al. Human adult neurogenesis: evidence and remaining questions. Stem Cell 23, 1-6 (2018).

2. Ernst, A. et al. Neurogenesis in the striatum of the adult human brain. Cell 156, 1072-1083 (2014).

3. Ernst, A. \& Frisén, J. Adult neurogenesis in humans- common and unique traits in mammals. PLoS Biol. 13, e1002045 (2015).

4. Bergmann, O. et al. The age of olfactory bulb neurons in humans. Neuron $\mathbf{7 4}$, 634-639 (2012).

5. Sanai, N. et al. Corridors of migrating neurons in the human brain and their decline during infancy. Nature 478, 382-386 (2011).

6. Silva-Vargas, V., Maldonado-Soto, A. R., Mizrak, D., Codega, P. \& Doetsch, F. Age-dependent niche signals from the choroid plexus regulate adult neural stem cells. Cell Stem Cell 19, 643-652 (2016).

7. Kalamakis, G. et al. Quiescence modulates stem cell maintenance and regenerative capacity in the aging brain. Cell 176, 1407-1419.e14 (2019).

8. Leeman, D. S. et al. Lysosome activation clears aggregates and enhances quiescent neural stem cell activation during aging. Science 359, 1277-1283 (2018).

9. Furutachi, S. et al. Slowly dividing neural progenitors are an embryonic origin of adult neural stem cells. Nat. Neurosci. 18, 657-665 (2015).

10. Fuentealba, L. C. et al. Embryonic origin of postnatal neural stem cells. Cell 161, 1644-1655 (2015).

11. Basak, O. et al. Troy+ brain stem cells cycle through quiescence and regulate their number by sensing niche occupancy. Proc. Natl Acad. Sci. USA 115, E610-E619 (2018).

12. Llorens-Bobadilla, E. et al. Single-cell transcriptomics reveals a population of dormant neural stem cells that become activated upon brain injury. Cell Stem Cell 17, 329-340 (2015).

13. Obernier, K. et al. Adult neurogenesis is sustained by symmetric self-renewa and differentiation. Cell Stem Cell 22, 221-234 (2018).

14. Zywitza, V., Misios, A., Bunatyan, L., Willnow, T. E. \& Rajewsky, N. Singlecell transcriptomics characterizes cell types in the subventricular zone and uncovers molecular defects impairing adult neurogenesis. Cell Rep. 25, 2457-2469 (2018)

15. Zelentsova, $K$. et al. Protein $S$ regulates neural stem cell quiescence and neurogenesis. Stem Cells 35, 679-693 (2017)

16. Borrett, M. J. et al. Single-cell profiling shows murine forebrain neural stem cells reacquire a developmental state when activated for adult neurogenesis. Cell Rep. 32, 108022 (2020).

17. Hirabayashi, Y. et al. Polycomb limits the neurogenic competence of neural precursor cells to promote astrogenic fate transition. Neuron 63, 600-613 (2009).

18. Nieto-González, J. L. et al. Loss of postnatal quiescence of neural stem cells through mTOR activation upon genetic removal of cysteine string protein- $\alpha$. Proc. Natl Acad. Sci. USA 116, 8000-8009 (2019).

19. Sueda, R., Imayoshi, I., Harima, Y. \& Kageyama, R. High Hes1 expression and resultant Ascll suppression regulate quiescent vs. active neural stem cells in the adult mouse brain. Genes Dev. 33, 511-523 (2019).

20. Otsuki, L. \& Brand, A. H. Dorsal-ventral differences in neural stem cell quiescence are induced by p57KIP2/Dacapo. Dev. Cell 49, 293-300.e3 (2019).

21. Otsuki, L. \& Brand, A. H. Cell cycle heterogeneity directs the timing of neural stem cell activation from quiescence. Science 360, 99-102 (2018).

22. Codega, P. et al. Prospective identification and purification of quiescent adult neural stem cells from their in vivo niche. Neuron 82, 545-559 (2014).

23. Furutachi, S., Matsumoto, A., Nakayama, K. I. \& Gotoh, Y. P57 controls adult neural stem cell quiescence and modulates the pace of lifelong neurogenesis. EMBO J. 32, 970-981 (2013).

24. Marchetti, B. et al. Parkinson's disease, aging and adult neurogenesis: $\mathrm{Wnt} / \beta$ catenin signalling as the key to unlock the mystery of endogenous brain repair. Aging Cell 19, 1-41 (2020). 
25. Nicaise, A. M., Willis, C. M., Crocker, S. J. \& Pluchino, S. Stem cells of the aging brain. Front. Aging Neurosci. 12, 1-23 (2020).

26. Belenguer, G. et al. Adult neural stem cells are alerted by systemic inflammation through TNF- $\alpha$ receptor signaling. Cell Stem Cell 28, 285-299.e9 (2021).

27. Azim, K. et al. Pharmacogenomic identification of small molecules for lineage specific manipulation of subventricular zone germinal activity. PLoS Biol. 15, 1-27 (2017).

28. Bragado Alonso, S. et al. An increase in neural stem cells and olfactory bulb adult neurogenesis improves discrimination of highly similar odorants. $E M B O$ J. 38, 1-13 (2019).

29. Van Den Berge, S. A. et al. The proliferative capacity of the subventricular zone is maintained in the parkinsonian brain. Brain 134, 3249-3263 (2011).

30. van Strien, M. E. et al. Isolation of neural progenitor cells from the human adult subventricular zone based on expression of the cell surface marker CD271. Stem Cells Transl. Med. 3, 470-480 (2014).

31. Donega, V. et al. Transcriptome and proteome profiling of neural stem cells from the human subventricular zone in Parkinson's disease. Acta Neuropathol. Commun. 7, 84 (2019).

32. Hashimshony, T., Wagner, F., Sher, N. \& Yanai, I. CEL-Seq: single-cell RNASeq by multiplexed linear amplification. Cell Rep. 2, 666-673 (2012).

33. Butler, A., Hoffman, P., Smibert, P., Papalexi, E. \& Satija, R. Integrating singlecell transcriptomic data across different conditions, technologies, and species. Nat. Biotechnol. 36, 411-420 (2018).

34. Marques, S. et al. Oligodendrocyte heterogeneity in the mouse juvenile and adult central nervous. Science 352, 1326 (2016).

35. Batista-Brito, R. et al. The cell-intrinsic requirement of Sox6 for cortical interneuron development. Neuron 63, 466-481 (2009).

36. Azim, E., Jabaudon, D., Fame, R. M. \& MacKlis, J. D. SOX6 controls dorsal progenitor identity and interneuron diversity during neocortical development. Nat. Neurosci. 12, 1238-1247 (2009).

37. Panman, L. et al. Sox 6 and Otx 2 control the specification of substantia nigra and ventral tegmental area dopamine neurons. Cell Rep. 8, 1018-1025 (2014).

38. Zhong, S. et al. A single-cell RNA-seq survey of the developmental landscape of the human prefrontal cortex. Nature 555, 524-528 (2018).

39. Jäkel, S. et al. Altered human oligodendrocyte heterogeneity in multiple sclerosis. Nature 566, 543-547 (2019).

40. Esteve, P., Crespo, I., Kaimakis, P., Sandonís, A. \& Bovolenta, P. Sfrp1 modulates cell-signaling events underlying telencephalic patterning, growth and differentiation. Cereb. Cortex 29, 1059-1074 (2019).

41. Shimogori, T., VanSant, J., Paik, E. \& Grove, E. A. Members of the Wnt, Fz, and Frp gene families expressed in postnatal mouse cerebral cortex. J. Comp. Neurol. 473, 496-510 (2004).

42. Liang, C.-J. et al. SFRPs are biphasic modulators of Wnt-signaling-elicited cancer stem cell properties beyond extracellular control. Cell Rep. 28, 1511-1525.e5 (2019).

43. Hawkshaw, N. J. et al. Identifying novel strategies for treating human hair loss disorders: Cyclosporine A suppresses the Wnt inhibitor, SFRP1, in the dermal papilla of human scalp hair follicles. PLoS Biol. 16, 1-17 (2018).

44. Kele, J. et al. SFRP1 and SFRP2 dose-dependently regulate midbrain dopamine neuron development in vivo and in embryonic stem cells. Stem Cells $\mathbf{3 0}$, 865-875 (2012).

45. Lange, C., Huttner, W. B. \& Calegari, F. Cdk4/CyclinD1 overexpression in neural stem cells shortens G1, delays neurogenesis, and promotes the generation and expansion of basal progenitors. Cell Stem Cell 5, 320-331 (2009).

46. Mizrak, D. et al. Single-cell analysis of regional differences in adult V-SVZ neural stem cell lineages. Cell Rep. 26, 394-406.e5 (2019).

47. Merkle, F. T., Mirzadeh, Z. \& Alvarez-buylla, A. Mosaic organization of neural stem cells in the adult brain. Science 317, 381-384 (2007).

48. Petratos, S. et al. Expression of the low-affinity neurotrophin receptor, p75NTR, is upregulated by oligodendroglial progenitors adjacent to the subventricular zone in response to demyelination. Glia 48, 64-75 (2004).

49. Yeung, M. S. Y. et al. Dynamics of oligodendrocyte generation and myelination in the human brain. Cell 159, 766-774 (2014).

50. Rueda-Carrasco, J. et al. Astrocyte to microglia cross-talk in acute and chronic neuroinflammation is shaped by SFRP1. bioRxiv https://doi.org/10.1101/ 2020.03.10.982579 (2020)

51. Esteve, P. et al. Elevated levels of Secreted-Frizzled-Related-Protein 1 contribute to Alzheimer's disease pathogenesis. Nat. Neurosci. 22, 1258-1268 (2019).

52. Jang, M. H. et al. Secreted frizzled-related protein 3 regulates activitydependent adult hippocampal neurogenesis. Cell Stem Cell 12, 215-223 (2013).

53. Kongkham, P. N. et al. The SFRP family of WNT inhibitors function as novel tumor suppressor genes epigenetically silenced in medulloblastoma. Oncogene 29, 3017-3024 (2010).
54. Dahl, E. et al. Frequent loss of SFRP1 expression in multiple human solid tumours: Association with aberrant promoter methylation in renal cell carcinoma. Oncogene 26, 5680-5691 (2007).

55. Suzuki, $H$. et al. Epigenetic inactivation of SFRP genes allows constitutive WNT signaling in colorectal cancer. Nat. Genet. 36, 417-422 (2004).

56. Bodine, P. V. N. et al. A small molecule inhibitor of the Wnt antagonist secreted frizzled-related protein-1 stimulates bone formation. Bone $\mathbf{4 4}$, 1063-1068 (2009).

57. Muraro, M. J. et al. A single-cell transcriptome atlas of the human pancreas. Cell Syst. 3, 385-394.e3 (2016).

58. Kase, Y., Otsu, K., Shimazaki, T. \& Okano, H. Involvement of p38 in agerelated decline in adult neurogenesis via modulation of Wnt signaling. Stem Cell Rep. 12, 1313-1328 (2019).

59. Bonnefont, J. et al. Cortical neurogenesis requires Bcl6-mediated transcriptional repression of multiple self-renewal-promoting extrinsic pathways. Neuron 103, 1096-1108.e4 (2019).

\section{Acknowledgements}

This work was supported by an Off-road grant (04510011910009) to V.D, a TAS-ZonMw grant (40-41400-98-16020) to E.M.H., by the MAXOMOD consortium, under the frame of E-Rare-3, the ERANET for Research on Rare Diseases (ERARE18-070) to R.J.P., and a Ministry of Science and Technology of China (MOST, 2016YFC1000500), a Theme-based Research Scheme (TRS, T13-602/21-N), a Health and Medical Research Fund (01120156) and CUHK Direct Grant (2019.052) to C.C.W. We are grateful to Peter Burbach for discussions and comments on the manuscript. We thank Christiaan van der Meer, Youri Adolfs, Roger Koot, and Nina Chu for technical support. The single-cell RNA sequencing was performed at Single Cell Discoveries by Judith Vivié and Mauro Muraro.

\section{Author contributions}

V.D. conceived and designed the study, performed and analyzed the single-cell RNAseq and mouse experiments. A.T.G. performed and analyzed in vitro experiments. J.A.S performed Luciferase reporter assay. R.E.D. performed FACS. C.C.W. provided fetal brain tissue. O.B. provided the protocol for single-cell sorting and performed alignment of the sequenced data. V.D. wrote the manuscript with input from E.M.H., R.J.P., O.B., and C.C.W. All authors revised and approved the manuscript.

\section{Competing interests}

The authors declare no competing interests.

\section{Additional information}

Supplementary information The online version contains supplementary material available at https://doi.org/10.1038/s41467-022-28626-9.

Correspondence and requests for materials should be addressed to Vanessa Donega or Elly M. Hol.

Peer review information Nature Communications thanks Bianca Marchetti and the other anonymous reviewers for their contribution to the peer review of this work.

Reprints and permission information is available at http://www.nature.com/reprints

Publisher's note Springer Nature remains neutral with regard to jurisdictional claims in published maps and institutional affiliations.

Open Access This article is licensed under a Creative Commons Attribution 4.0 International License, which permits use, sharing, adaptation, distribution and reproduction in any medium or format, as long as you give appropriate credit to the original author(s) and the source, provide a link to the Creative Commons license, and indicate if changes were made. The images or other third party material in this article are included in the article's Creative Commons license, unless indicated otherwise in a credit line to the material. If material is not included in the article's Creative Commons license and your intended use is not permitted by statutory regulation or exceeds the permitted use, you will need to obtain permission directly from the copyright holder. To view a copy of this license, visit http://creativecommons.org/ licenses/by/4.0/.

(C) The Author(s) 2022 